\title{
Exploring the nature of chiral phase transition in two-flavor QCD using extra heavy quarks
}

\author{
Shinji Ejiri, ${ }^{1}$ Ryo Iwami, ${ }^{2}$ and Norikazu Yamada ${ }^{3,4}$ \\ 1 Department of Physics, Niigata University, Niigata 950-2181, Japan \\ 2 Graduate School of Science and Technology, \\ Niigata University, Niigata 950-2181, Japan \\ ${ }^{3}$ KEK Theory Center, Institute of Particle and Nuclear Studies, \\ High Energy Accelerator Research Organization (KEK), Tsukuba 305-0801, Japan \\ 4 School of High Energy Accelerator Science, \\ SOKENDAI (The Graduate University for Advanced Studies), Tsukuba 305-0801, Japan
}

(Dated: January 4, 2018)

\begin{abstract}
Chiral phase transition of two-flavor QCD at finite quark masses is known to be a crossover except near the chiral limit, but it can turn to a first order transition when adding many extra flavors. This property is used to explore the nature of the phase transition of massless two-flavor QCD using lattice numerical simulations. The extra heavy flavors being incorporated in the form of the hopping parameter expansion through the reweighting, the number of the extra flavors and their masses appear only in a single parameter, defined by $h$. We determine the critical value of $h$, at which the first order and the crossover regions are separated, and examine its dependence on the two-flavor mass. The lattice calculations are carried out at $N_{t}=4$, and show that the critical value of $h$ does not depend on the two-flavor mass in the range we have studied $\left(0.46 \leq m_{\pi} / m_{\rho} \leq 0.66\right)$ and appears to remain finite and positive in the chiral limit, suggesting that the phase transition of massless two-flavor QCD is of second order.
\end{abstract}

PACS numbers: 11.15.Ha, 12.38.Gc, 12.38.Mh, 12.60.Nz 


\section{INTRODUCTION}

Quantum chromodynamics (QCD) shows a variety of phases, and in passing over a phase boundary one would encounter either first (discontinuous) or second order (continuous) transition, depending on temperature, density, quark masses, the number of flavors, etc. Chiral phase transition of QCD with two massless quarks at the vanishing chemical potential has been studied with various approaches for a long time, since it provides us with a solid basis in the study of $2+1$-flavor QCD in the real world. Nevertheless, the nature of the transition of this relatively simple system is yet ambiguous, and is counted as one of the longstanding problems.

Based on the universality argument and the results of the leading order $\epsilon$ expansion, Pisarski and Wilczek analyzed the renormalization group (RG) flow of the three-dimensional scalar field theory, which shares the same internal symmetry with massless QCD around the critical temperature $\left(T_{c}\right)$, and pointed out that, in the two-flavor case, the order of the transition could crucially depend on the presence (or the absence) of the flavor singlet axial $\left(U_{A}(1)\right)$ symmetry at $T_{c}$ [1]. If $U_{A}(1)$ symmetry is largely violated at $T_{c}$, the second order phase transition with the $O(4)$ scaling becomes possible although not mandatory. On the other hand, when the symmetry is effectively and fully restored at $T_{c}$, the leading order calculation of the $\epsilon$ expansion suggests no infrared fixed point (IRFP), and hence the second order phase transition is excluded. But, later, further studies using different advanced techniques found evidence of IRFP, and the confirmation of the presence of the IRFP is under active investigation [2-5]. Thus, the transition in this case again can be either of first or second order. Recently, a novel possibility is pointed out, following the RG flow analysis: in the presence of small but finite $U_{A}(1)$ symmetry breaking, the system may undergo the second order transition with the $O(4)$ scaling but one of the critical exponents related to the

scaling dimension of the leading irrelevant operator is different from that of the $O(4)$ [6], although again the second order transition is not mandatory.

Numerical simulations of QCD on the lattice can, in principle, determine the order of the transition as well as the universality class, to which massless two-flavor QCD belongs, by performing the scaling study [7-15]. However, it is not easy to keep all the systematic and statistical uncertainties under control in the chiral limit due to large computational costs. Furthermore, it appears that, in practice, the standard scaling study may not be 
efficient enough to distinguish the first and the second order transitions because the scaling functions are similar between the $Z_{2}$ and $O(4)$ universality classes [16]. With lattice QCD simulations, one can also study the presence (or absence) of $U_{A}(1)$ symmetry through the Dirac spectrum. For recent progress, see, for example, Refs. [17-20].

Clarifying this point is important not only for understanding the QCD phase diagram but also for the scenario of the axion dark matter. The axion abundance is essentially determined by the temperature dependence of the topological susceptibility, $\chi_{t}(T)$, which vanishes when $U_{A}(1)$ symmetry is fully and effectively restored. If $\chi_{t}$ vanishes very rapidly right above $T_{c}$, too many axions would be produced, and the axion dark matter scenario becomes hard or is even excluded [21], depending on how rapidly it vanishes. The lattice studies to test the axion dark matter scenario has recently begun in the quenched approximation [21-23].

In this paper, we follow the approach proposed in Ref. [24], in which the phase transition of two-flavor QCD is studied by adding many extra heavy quarks. We call this the many flavor approach. The transition of two-flavor QCD at a finite quark mass is known to be a crossover, but it can turn to a first order transition when adding many extra flavors. This property is used to explore the nature of the phase transition of massless two-flavor QCD. The extra quarks are incorporated in the form of the hopping parameter expansion (HPE) through the reweighting. Then the number of the extra flavors $\left(N_{f}\right)$ and their mass parameter $\left(\kappa_{h}\right)$ appear in a single parameter

$$
h=2 N_{f}\left(2 \kappa_{h}\right)^{N_{t}}
$$

where $N_{t}$ denotes the number of lattice sites in the temporal direction. We determine the critical value of the parameter $\left(h_{c}\right)$ at which the first order and the crossover regions are separated, and examine its dependence on the two-flavor mass. The order of the transition for a given $h$ is discriminated by the shape of the constraint effective potential at $T_{c}$, which is constructed from the probability distribution function (PDF) for a generalized plaquette. Namely, it is discriminated by whether the potential at $T_{c}$ is in single- or double-well shape. It is important to note that, in the determination of $h_{c}$ in this approach, the convergence of the HPE is not the matter since we can consider arbitrary small $\kappa_{h}$ by considering arbitrary large $N_{f}$ as seen from Eq.(1).

We perform an exploratory study on $N_{t}=4$ and try to see how $h_{c}$ depends on the twoflavor mass. Then, $h_{c}$ is found to stay constant against the change of the two-flavor mass 
in the range we have studied and to remain positive and finite in the chiral limit. Since the two-flavor system is equivalent to the $2+N_{f}$-flavor system with $h=0$, our result suggests that massless two-flavor QCD belongs to the region of second order phase transition. This kind of extension of QCD is useful also for the study of the phase structure in the presence of finite chemical potential [24]. The similar approach but introducing a finite imaginary chemical potential is taken in Ref. [15].

The paper is organized as follows. After the central idea of many flavor approach is explained in Sec. II, the method is described in detail in Sec. III. The lattice setup and the main part of this paper are given in Sec. IV. In Sec. V, two independent analyses are

performed for the consistency check. Finally, the conclusion and perspectives are stated in Sec. VI. The preliminary result of this work is available in Ref. [25].

\section{MANY FLAVOR APPROACH}

The central idea of many flavor approach is outlined. Figure 1 (a) shows the so-called Columbia plot for $2+1$-flavor QCD [26], which summarizes the present knowledge on the mass dependent nature of the phase transition of QCD as a function of $m_{u d}$ and $m_{s}$. The physical point is believed to be located in the crossover region $[13,27]$. The plot tells us that there are two distinct first order regions lying around the quenched limit $\left(m_{u d}=m_{s}=\infty\right)$ and the chiral limit of three-flavor QCD $\left(m_{u d}=m_{s}=0\right)$, respectively. In what follows, we focus on the latter.

Our interest is in whether the massless two-flavor QCD point $\left(m_{u d}=0\right.$ and $\left.m_{s}=\infty\right)$ is inside the first order region or not and hence in the shape of the first order region. If it ends at a finite $m_{s}$, called the tricritical point, massless two-flavor QCD is on the line of the second order phase transition (the solid curve on the $m_{u d}=0$ line). On the other hand, if the first order region extends to $m_{s}=\infty$, massless two-flavor QCD should undergo the first order transition (i.e. the dotted curve near the $m_{u d}=0$ line). In either case, if we could resolve which of the solid or dotted curves is realized, we would be able to answer the question. However, it is difficult to trace the critical line for the $2+1$-flavor case, because the critical line is located in the small $m_{s}$ region when $m_{u d}$ is moderately small $[13,28,29]$.

The situation will change in $2+N_{f}$-flavor QCD as shown in Fig. 1 (b). The bottom-right corner of Fig. 1 (b) is of first order for $N_{f} \geq 3$. The critical line is expected to move upward 


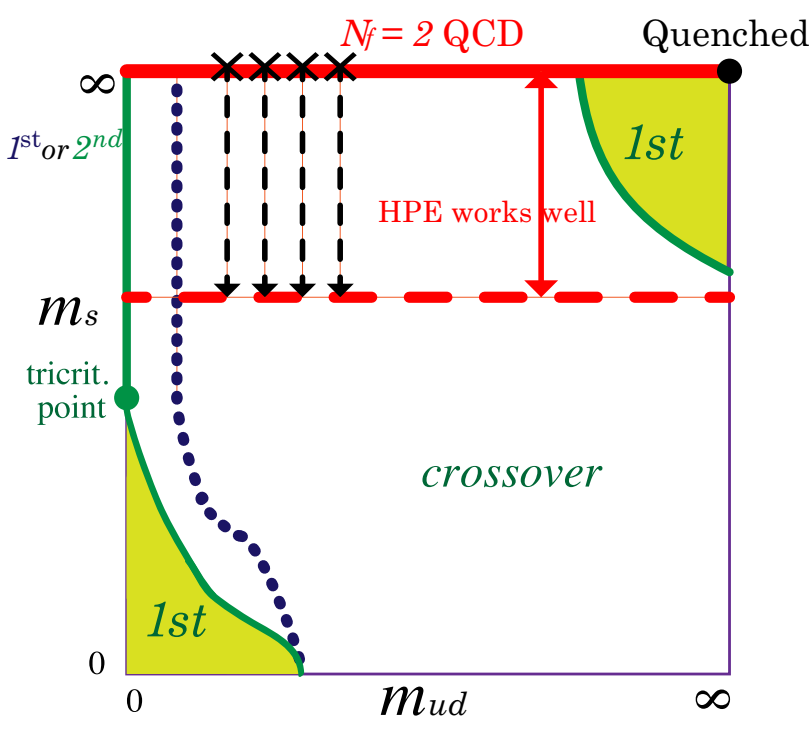

(a) 2+1 flavor QCD

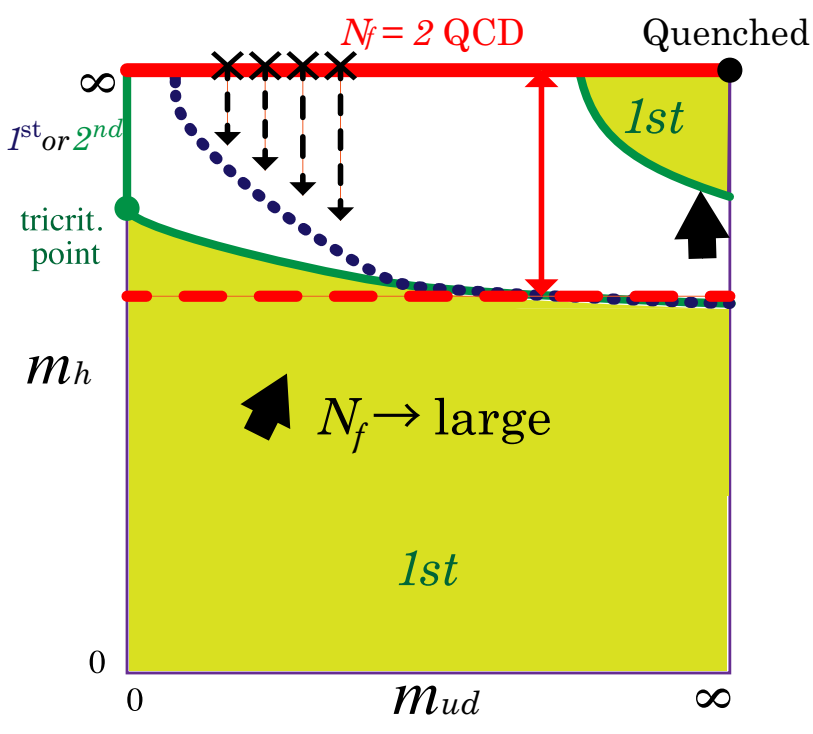

(b) $2+N_{f}$ flavor QCD

FIG. 1: Basic idea of "many flavor approach".

as $N_{f}$ increases, and for sufficiently large $N_{f}$ it could enter the region where the hopping parameter expansion from the static limit (crosses at $m_{h}=\infty$ ) works well (above the dashed line). Then, one should be able to easily identify how the critical line runs as a function of $m_{u d}$. If the critical heavy mass $m_{h}^{c}\left(m_{u d}\right)$ remains finite in the $m_{u d} \rightarrow 0$ limit, it immediately means that massless two-flavor QCD corresponding to the point $\left(m_{u d}, m_{h}\right)=(0, \infty)$ is the outside of the first order region. An important remark is that, in the limit of $m_{s} \rightarrow \infty$ or $m_{h} \rightarrow \infty$, both $2+1$ - and $2+N_{f}$-flavor QCD end up with the same theory, which we want to study. Thus, the original question is simplified to the one whether the critical heavy mass in the chiral limit stays finite or not.

While most of the current knowledge on the shape of the first order region are only qualitative, in a context of the Taylor-expanded reweighting method [30] we can derive a solid statement about the slope at $m_{u d}=m_{h}$ if the same quark action is employed for two- and $N_{f}$-flavors. Suppose that $m_{u d}=m+\Delta m_{u d}$ and $m_{h}=m+\Delta m_{h}$ and expand the logarithm of the quark determinants in terms of $\Delta m_{u d}$ and $\Delta m_{h}$. Then, one will be aware that the partition function does not change as long as $\Delta m_{h}=-2 \Delta m_{u d} / N_{f}$ and hence that physics is identical along the line of slope $-2 / N_{f}$ near $m_{u d}=m_{h}$ line in the $\left(m_{u d}, m_{h}\right)$ plane [31]. This means that the critical line in $2+N_{f}$-flavor QCD crosses the line 
of $m_{u d}=m_{h}$ with a slope milder than the $2+1$ flavor case.

In Ref [24], it is demonstrated that by adding extra flavors the end point (or the critical line) indeed enters the region reachable by the hopping parameter expansion. In this paper, we examine the light quark mass dependence of the end point. As we will explain below, $m_{h}^{c}\left(m_{u d}\right)$ seems to remain finite in the chiral limit of $m_{u d}$, suggesting the phase transition of massless two-flavor QCD is of second order.

\section{CALCULATIONAL METHOD}

We first generate two-flavor configurations at finite temperatures following the standard hybrid Monte Carlo method. Using the hopping parameter expansion and the reweighting method, we incorporate extra $N_{f}$ flavors of heavy quarks into those configurations, and measure the probability distribution function for a generalized plaquette to construct the constraint effective potential for $2+N_{f}$-flavor QCD.

After the HPE, the hopping parameter for heavy quarks $\kappa_{h}$ and $N_{f}$ appear only in a single parameter, $h$ [see Eq. (15)]. If the parameter $h$ is in the crossover region, the effective potential should take a single-well shape at the pseudocritical temperature, $T_{p c}$. On the other hand, when the parameter $h$ enters the first order region, a double-well shape should emerge at $T_{c}$. By scanning $h$, we determine the critical value $h_{c}$, at which the first order and the crossover regions are separated. The critical value $h_{c}$ is determined at four values of twoflavor mass to see the light quark mass dependence of $h_{c}$. In the following, the calculational procedure is described in detail.

The PDF was introduced in Refs. [32, 33] and has been extensively used in various fields to study the critical properties of various materials [34] or the phase diagrams of QCD [35]. In our study of $2+N_{f}$-flavor QCD, the PDF $w$ for a quantity $\hat{X}$ is defined by

$$
w\left(X ; \beta, \kappa_{l}, \kappa_{h}, N_{f}\right)=\int \mathcal{D} U \delta(X-\hat{X})\left[\operatorname{det} M\left(\kappa_{h}\right)\right]^{N_{f}} e^{-S_{\text {gauge }}(\beta)-S_{\text {light }}\left(\kappa_{l}\right)},
$$

where $S_{\text {gauge }}(\beta)$ and $S_{\text {light }}\left(\kappa_{l}\right)$ are the lattice actions for the gauge field and two flavors of light quarks, respectively. $\beta=6 / g_{0}^{2}$ is the simulation parameter setting the temperature through the lattice spacing, and $\kappa_{l}$ is the light quark mass parameter. Note that the method described below works for any kinds of light quark action unless it contains $\beta$ dependent coefficients. The action for the $N_{f}$ extra flavors is written in the determinant form in Eq. (2), 
where $M\left(\kappa_{h}\right)$ is the lattice Dirac operator for heavy quarks with a mass parameter $\kappa_{h}$. A quantity to be constrained, $\hat{X}$, is basically arbitrary, but the order parameter would be the most natural choice if it is available. In this paper, following previous works [24,35-37], $\hat{X}$ is chosen to be the generalized plaquette

$$
\hat{P}=c_{0} \hat{W}_{P}+2 c_{1} \hat{W}_{R}
$$

where $\hat{W}_{P}$ and $\hat{W}_{R}$ denote the averaged plaquette and rectangle, respectively, and $c_{0}$ and $c_{1}$ satisfying $c_{0}=1-8 c_{1}$ are the improvement coefficients for lattice gauge action. In terms of $\hat{P}$, the gauge action is written as

$$
S_{\text {gauge }}(\beta)=-6 N_{\text {site }} \beta \hat{P},
$$

where $N_{\text {site }}=N_{\mathrm{s}}^{3} \times N_{t}$ represents the number of sites in four-dimensional lattice volume. Our main analysis is carried out with $\hat{X}=\hat{P}$, but the calculation for $\hat{X}=\hat{L}$ with $\hat{L}$ the real part of the Polyakov loop averaged over spatial sites is also performed as a consistency check (see Sec. VA). Choosing $\hat{X}=\hat{P}$ brings a great simplification in the numerical analysis as explained below. In principle, we could choose other quantities, e.g. the chiral condensate, to be $\hat{X}$. But, whenever a quantity other than $\hat{P}$ is chosen, we lose not only the advantage for $\hat{X}=\hat{P}$ but also the accuracy in the results as demonstrated in Sec. VA for $\hat{X}=\hat{L}$.

With the PDF thus obtained, the constraint effective potential $V$ is calculated by

$$
V\left(X ; \beta, \kappa_{l}, \kappa_{h}, N_{f}\right)=-\ln w\left(X ; \beta, \kappa_{l}, \kappa_{h}, N_{f}\right)
$$

In practice, the PDF is not directly accessible, and hence we instead calculate the histogram defined by

$$
H\left(X ; \beta, \kappa_{l}, \kappa_{h}, N_{f}\right)=\frac{w\left(X ; \beta, \kappa_{l}, \kappa_{h}, N_{f}\right)}{Z\left(\beta, \kappa_{l}, \kappa_{h}, N_{f}\right)},
$$

where $Z(\beta)$ is the partition function. Note that $Z(\beta)$ is not calculable, but its ratio at two different $\beta$ values is calculable [38].

In order to see the shape of the potential, we need to calculate the potential over a certain range of $X$. However, a simulation at a single $\beta$ provides the potential only in a limited range of $X$. Thus, the potential calculated at a certain $\beta$ needs to be translated to that at other $\beta$. We call the temperature, at which we want to calculate the potential, the reference 
temperature (or $\beta_{\text {ref }}$ ). Then, the potential is calculated as

$$
\begin{aligned}
& V\left(X ; \beta_{\mathrm{ref}}, \kappa_{l}, \kappa_{h}, N_{f}\right)+\ln Z\left(\beta^{*}, \kappa_{l}^{\prime}, \kappa_{h}^{\prime}, N_{f}^{\prime}\right) \\
= & -\ln H\left(X^{\prime} ; \beta^{\prime}, \kappa_{l}^{\prime}, \kappa_{h}^{\prime}, N_{f}^{\prime}\right)-\ln \left(\frac{w\left(X^{\prime \prime} ; \beta^{\prime \prime}, \kappa_{l}^{\prime \prime}, \kappa_{h}^{\prime \prime}, N_{f}^{\prime \prime}\right)}{w\left(X^{\prime} ; \beta^{\prime}, \kappa_{l}^{\prime}, \kappa_{h}^{\prime}, N_{f}^{\prime}\right)}\right)-\ln \left(\frac{w\left(X ; \beta_{\mathrm{ref}}, \kappa_{l}, \kappa_{h}, N_{f}\right)}{w\left(X^{\prime \prime} ; \beta^{\prime \prime}, \kappa_{l}^{\prime \prime}, \kappa_{h}^{\prime \prime}, N_{f}^{\prime \prime}\right)}\right) \\
& -\ln \frac{Z\left(\beta^{\prime}, \kappa_{l}^{\prime}, \kappa_{h}^{\prime}, N_{f}^{\prime}\right)}{Z\left(\beta^{*}, \kappa_{l}^{\prime}, \kappa_{h}^{\prime}, N_{f}^{\prime}\right)} .
\end{aligned}
$$

All the intermediate quantities such as $\beta^{\prime}, X^{\prime}, \kappa_{l}^{\prime}, \kappa_{h}^{\prime}$ and $N_{f}^{\prime}$ are arbitrary as well as $\beta^{*}$. For a practical reason, we take

$$
X^{\prime}=X^{\prime \prime}=X, \quad \beta^{\prime \prime}=\beta_{\text {ref }}, \quad \kappa_{l}^{\prime}=\kappa_{l}^{\prime \prime}=\kappa_{l}, \quad \kappa_{h}^{\prime}=\kappa_{h}^{\prime \prime}=0 \quad N_{f}^{\prime}=N_{f}^{\prime \prime}=0,
$$

and $\beta^{*}$ is chosen to be the vicinity of $\beta_{\text {ref }}$.

In the following, we take $\hat{X}=\hat{P}$, then the effective potential is simplified as

$$
V\left(P ; \beta_{\text {ref }}, \kappa_{l}, \kappa_{h}, N_{f}\right)+\ln Z\left(\beta^{*}, \kappa_{l}^{\prime}, \kappa_{h}^{\prime}, N_{f}^{\prime}\right)=V_{\text {light }}\left(P ; \beta_{\text {ref }}, \kappa_{l}\right)-\ln R\left(P ; \beta_{\text {ref }}, \kappa_{l}, \kappa_{h}, N_{f}\right) .
$$

The first term is defined by

$$
V_{\text {light }}\left(P ; \beta_{\text {ref }}, \kappa_{l}\right)=-\ln H\left(P ; \beta, \kappa_{l}, 0,0\right)-6 N_{\text {site }}\left(\beta_{\text {ref }}-\beta\right) P-\ln \frac{Z\left(\beta, \kappa_{l}, 0,0\right)}{Z\left(\beta^{*}, \kappa_{l}, 0,0\right)}
$$

and represents the constraint effective potential for two flavors alone. The second term of Eq. (9) is defined by

$$
\begin{aligned}
R\left(P ; \beta_{\text {ref }}, \kappa_{l}, \kappa_{h}, N_{f}\right) & =\left\langle\left[\operatorname{det} M\left(\kappa_{h}\right)\right]^{N_{f}}\right\rangle_{P: \text { fixed },\left(\beta_{\text {ref }}, \kappa_{l}\right)}, \\
\langle\cdots\rangle_{P: \text { fixed },\left(\beta_{\text {ref },}, \kappa_{l}\right)} & \equiv \frac{\langle\delta(P-\hat{P}) \cdots\rangle_{\left(\beta_{\text {ref }}, \kappa_{l}\right)}}{\langle\delta(P-\hat{P})\rangle_{\left(\beta_{\text {ref }}, \kappa_{l}\right)}}
\end{aligned}
$$

where $\langle\cdots\rangle_{\left(\beta, \kappa_{l}\right)}$ denotes the ensemble average over two-flavor configurations generated with $\beta$ and $\kappa_{l}$. It is important to note that, separating the effective potential into the two-flavor part and the extra heavy part as in Eq. (9), the latter becomes independent of $\beta_{\text {ref }}$. The reason is as follows. Due to the operator $\delta(P-\hat{P})$, the factor of $\exp \left(6 N_{\text {site }} \beta_{\text {ref }} P\right)$ comes out of the brackets in Eq. (12). Since this factor cancels between the numerator and the denominator in Eq. (12), $\beta_{\text {ref }}$ dependence disappears. This simplification takes place only for $\hat{X}=\hat{P}$. 
For the extra heavy quarks, we employ the unimproved Wilson fermion because it suffices for the present purpose. For sufficiently small $\kappa_{h}$, the determinant in Eq. (11) can be approximated by the leading order of the HPE as

$$
\begin{aligned}
\ln \left[\operatorname{det} M\left(\kappa_{h}\right)\right]^{N_{f}} & \approx N_{f}\left(288 N_{\text {site }} \kappa_{h}^{4} \hat{W}_{P}+12 N_{s}^{3}\left(2 \kappa_{h}\right)^{4} \hat{L}\right) \\
& =6 N_{s}^{3} h \hat{Y} \\
& =9 N_{\text {site }} \frac{h}{c_{0}} \hat{P}+6 N_{s}^{3} h \hat{Z},
\end{aligned}
$$

where the following quantities have been introduced:

$$
\begin{aligned}
h & =2 N_{f}\left(2 \kappa_{h}\right)^{4}, \\
\hat{Y} & =6 \hat{W}_{P}+\hat{L}, \\
\hat{Z} & =-\frac{12 c_{1}}{c_{0}} \hat{W}_{R}+\hat{L} .
\end{aligned}
$$

Here and hereafter, $N_{t}=4$ is assumed because we take that value in numerical simulations, but the extension to other values of $N_{t}$ is straightforward though the expression becomes complicated. Then, $\ln R$ in Eq. (9) can be approximated as

$$
\begin{aligned}
\ln R\left(P ; \kappa_{l}, h\right) & \approx \ln \left\langle\exp \left(6 N_{s}^{3} h \hat{Y}\right)\right\rangle_{P: \text { fixed },\left(\beta, \kappa_{l}\right)} \\
& =9 N_{\text {site }} \frac{h}{c_{0}} P+\ln R^{\prime}\left(P ; \kappa_{l}, h\right)
\end{aligned}
$$

where

$$
\ln R^{\prime}\left(P ; \kappa_{l}, h\right)=\ln \left\langle\exp \left(6 N_{s}^{3} h \hat{Z}\right)\right\rangle_{P: \text { fixed, }\left(\beta, \kappa_{l}\right)}
$$

Although Eqs. (18) and (19) are algebraically identical, the equality is not necessarily trivial in numerical data because the $\delta$ function is approximated by

$$
\delta(x) \approx 1 /(\Delta \sqrt{\pi}) \exp \left[-(x / \Delta)^{2}\right]
$$

Then, the difference can arise when

$$
\left\langle e^{6 N_{s}^{3} h \hat{Y}} \exp \left[-(P-\hat{P})^{2} / \Delta^{2}\right]\right\rangle_{\left(\beta, \kappa_{l}\right)}-e^{9 N_{\text {site }} \frac{h}{c_{0}} P}\left\langle e^{6 N_{s}^{3} h \hat{Z}} \exp \left[-(P-\hat{P})^{2} / \Delta^{2}\right]\right\rangle_{\left(\beta, \kappa_{l}\right)} \neq 0,
$$

which should vanish for sufficiently small $\Delta$, but then the statistical error will enlarge. In the following analysis, both expressions are examined to check the consistency. 
It is important to note that, after the HPE, the number of extra heavy flavors $\left(N_{f}\right)$ and their mass parameter $\left(\kappa_{h}\right)$ appear only in a single parameter $h$, Eq. (15). Because of this, $\kappa_{h}$ and $N_{f}$ have been replaced by $h$ in the arguments of $R$ and $R^{\prime}$, and our purpose turns to finding the critical value of $h, h_{c}$. It should be also noted that the second derivatives of Eqs. (18) and (20) with regard to $P$ are identical because the difference is proportional to $P$.

One side remark is below. Thus far, we have restricted the extra heavy quarks to be degenerate. But the extension to the nondegenerate case is straightforward by interpreting $h$ as $h=2 \sum_{f=1}^{N_{f}}\left(2 \kappa_{h}\right)^{N_{t}}$. In the following, we only consider the degenerate case for simplicity.

Choosing $\hat{X}=\hat{P}$ significantly simplifies the procedure to find the critical value of $h[24,36]$ as follows. We are interested in the shape of the potential at the (pseudo)critical temperature, which requires $\beta_{\text {ref }}$ to be tuned to its (pseudo)critical value, $\beta_{c}$ (or $\beta_{p c}$ ). This tuning can be totally skipped if we look at the curvature, i.e. the second derivative of the potential with respect to $P$, because it is independent of $\beta_{\text {ref. }} R$ is independent of $\beta_{\text {ref }}$ as stated above. $V_{\text {light }}$ depends on $\beta_{\text {ref }}$, but its second derivative does not as explained below.

The finite temperature transition of two-flavor QCD is always a crossover for the twoflavor masses adopted in this paper. Then, at any temperatures, the shape of the PDF (or equivalently histogram) for $\hat{P}$ in two-flavor QCD can be well approximated, around the peak, by Gaussian form,

$$
\left.w\left(P ; \beta, \kappa_{l}, 0,0\right)\right|_{P \sim \bar{P}\left(\beta, \kappa_{l}\right)} \propto \exp \left[-\frac{6 N_{\text {site }}\left(P-\bar{P}\left(\beta, \kappa_{l}\right)\right)^{2}}{2 \chi_{P}\left(\beta, \kappa_{l}\right)}\right],
$$

where $\bar{P}\left(\beta, \kappa_{l}\right)=\langle\hat{P}\rangle_{\beta, \kappa_{l}}$ is the average of generalized plaquette at $\beta$ and $\kappa_{l}$, and $\chi_{P}$ is the susceptibility of $P$, given by

$$
\chi_{P}\left(\beta, \kappa_{l}\right)=6 N_{\text {site }}\left\langle\left(\hat{P}-\bar{P}\left(\beta, \kappa_{l}\right)\right)^{2}\right\rangle_{\beta, \kappa_{l}} .
$$

Substituting this into Eq. (10) yields, up to a constant shift,

$$
\left.V_{\text {light }}\left(P ; \beta_{\text {ref }}, \kappa_{l}\right)\right|_{P \sim \bar{P}\left(\beta, \kappa_{l}\right)}=\frac{6 N_{\text {site }}\left(P-\bar{P}\left(\beta, \kappa_{l}\right)\right)^{2}}{2 \chi_{P}\left(\beta, \kappa_{l}\right)}-6\left(\beta_{\text {ref }}-\beta\right) N_{\text {site }} P \text {. }
$$

Then, the first and second derivatives are given by

$$
\begin{aligned}
\left.\frac{d V_{\text {light }}\left(P ; \beta_{\text {ref }}, \kappa_{l}\right)}{d P}\right|_{P \sim \bar{P}\left(\beta, \kappa_{l}\right)} & =\frac{6 N_{\text {site }}\left(P-\bar{P}\left(\beta, \kappa_{l}\right)\right)}{\chi_{P}\left(\beta, \kappa_{l}\right)}-6\left(\beta_{\text {ref }}-\beta\right) N_{\text {site }}, \\
\left.\frac{d^{2} V_{\text {light }}\left(P ; \kappa_{l}\right)}{d P^{2}}\right|_{P \sim \bar{P}\left(\beta, \kappa_{l}\right)} & =\frac{6 N_{\text {site }}}{\chi_{P}\left(\beta, \kappa_{l}\right)} .
\end{aligned}
$$


Thus, we can calculate the curvature of the two-flavor part by collecting $\chi_{P}\left(\beta, \kappa_{l}\right)$ obtained at various $\beta$. Importantly, Eq. (26) is independent of $\beta_{\text {ref }}$. In summary, the curvature of the total effective potential

$$
\frac{d^{2} V\left(P ; \beta_{\mathrm{ref}}, \kappa_{l}, h\right)}{d P^{2}}=\frac{d^{2} V_{\text {light }}\left(P ; \beta_{\mathrm{ref}}, \kappa_{l}\right)}{d P^{2}}-\frac{d^{2} \ln R\left(P ; \kappa_{l}, h\right)}{d P^{2}},
$$

is independent of $\beta_{\text {ref. }}$.

The procedure to identify $h_{c}$ in the chiral limit of two flavors goes as follows. At $h=0$, the contribution of the extra heavy flavors is trivially zero, and the system is reduced to two-flavor QCD, where the transition is a crossover. Therefore, the second derivative of the potential is always positive. As $h$ is increased from zero, the minimum of the curvature takes zero at some point, which gives $h_{c}$. In this procedure, one needs not tune $\beta_{\mathrm{ref}}$ to $\beta_{\mathrm{pc}}$ or $\beta_{\mathrm{c}}$, because the curvature is independent of the temperature or $\beta_{\text {ref }}$. This simplification does not occur in general, and one such example is explicitly shown in Sec. V A. By looking at the light quark mass dependence of $h_{c}\left(\kappa_{l}\right)$, we try to extract $h_{c}$ in the chiral limit.

\section{NUMERICAL RESULTS}

\section{A. Simulation parameters}

Following Ref. [39], we take the Iwasaki gauge action $\left(c_{1}=-0.331\right)$ and the $O(a)$ improved Wilson fermion action with the perturbatively improved $c_{\mathrm{sw}}$ for two flavors of light quarks. Simulations are performed on $N_{\text {site }}=16^{3} \times 4$ lattices with 25 to $32 \beta$ values at each of four $\kappa_{l}$, and 10,000 to 40,000 trajectories have been accumulated at each simulation point. Four light quark masses are ranging from $\kappa_{l}=0.145$ to 0.1505 . Figure 2 shows the histogram of the generalized plaquette.

At the pseudocritical point $\beta_{\mathrm{pc}}$ for each $\kappa_{l}$, we carried out zero temperature simulations on $16^{3} \times 32$ lattices to find the mass ratio of pseudoscalar and vector mesons, $m_{\pi} / m_{\rho}$, and the quark mass defined through the partially conserved axialvector current (PCAC),

$$
a m_{\mathrm{pcac}}=\frac{\left\langle\sum_{\vec{x}}\left(A_{4}\left(N_{t} / 2+1, \vec{x}\right)-A_{4}\left(N_{t} / 2-1, \vec{x}\right)\right) P(0)\right\rangle}{4\left\langle\sum_{\vec{x}} P\left(N_{t} / 2, \vec{x}\right) P(0)\right\rangle},
$$

where $A_{4}(x)$ and $P(x)$ are the flavor nonsinglet, local axialvector and pseudoscalar operator, respectively, and $N_{t}=32$. Then, the four values of $\kappa_{l}$ in this paper turn out to cover $0.46<m_{\pi} / m_{\rho}<0.66$, or $0.019<a m_{\text {pcac }}<0.054$. These results are tabulated in Table I. 

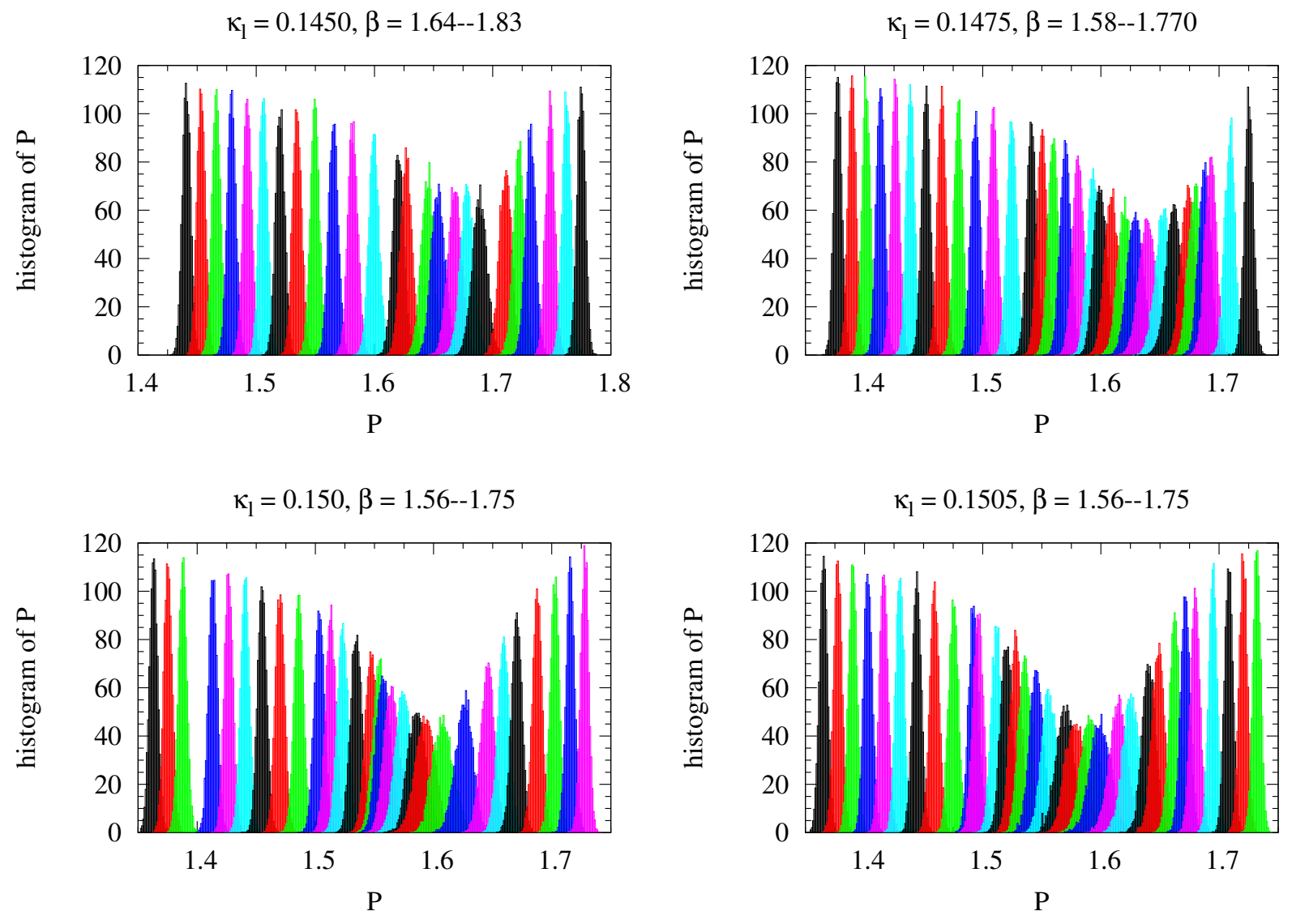

FIG. 2: Histogram of the generalized plaquette at four values of $\kappa_{l}$.

\begin{tabular}{cc|ccccc}
$\kappa_{l}$ & $c_{\mathrm{sw}}$ & $\beta_{\mathrm{pc}}$ & $a m_{\pi}$ & $a m_{\rho}$ & $m_{\pi} / m_{\rho}$ & $a m_{\mathrm{pcac}}$ \\
\hline 0.1450 & 1.650 & 1.778 & $0.779(1)$ & $1.172(2)$ & $0.665(33)$ & $0.0535(1)$ \\
0.1475 & 1.677 & 1.737 & $0.651(1)$ & $1.130(5)$ & $0.576(28)$ & $0.0350(2)$ \\
0.1500 & 1.707 & 1.691 & $0.514(2)$ & $1.099(10)$ & $0.468(24)$ & $0.0202(2)$ \\
0.1505 & 1.712 & 1.681 & $0.495(2)$ & $1.082(13)$ & $0.458(23)$ & $0.0186(2)$
\end{tabular}

TABLE I: Simulation parameters $\left(\kappa_{l}\right.$ and $\left.c_{\mathrm{sw}}\right)$ and the pseudocritical $\beta\left(\beta_{\mathrm{pc}}\right)$ in two-flavor QCD, determined from the peak of the susceptibility for the generalized plaquette. $m_{\pi} / m_{\rho}$ and other quantities are determined on a $16^{3} \times 32$ lattice at $\beta_{\mathrm{pc}}$ for each $\kappa_{l}$.

\section{B. Main results}

We present the numerical results for the two terms in the right-hand side of Eq. (27) separately, and focus on the second term first. With the approximated $\delta$ function (21), 


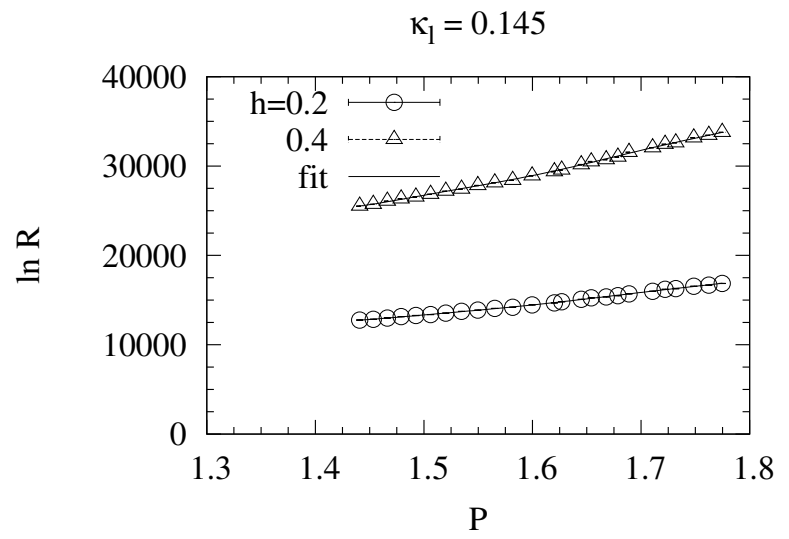

(a)

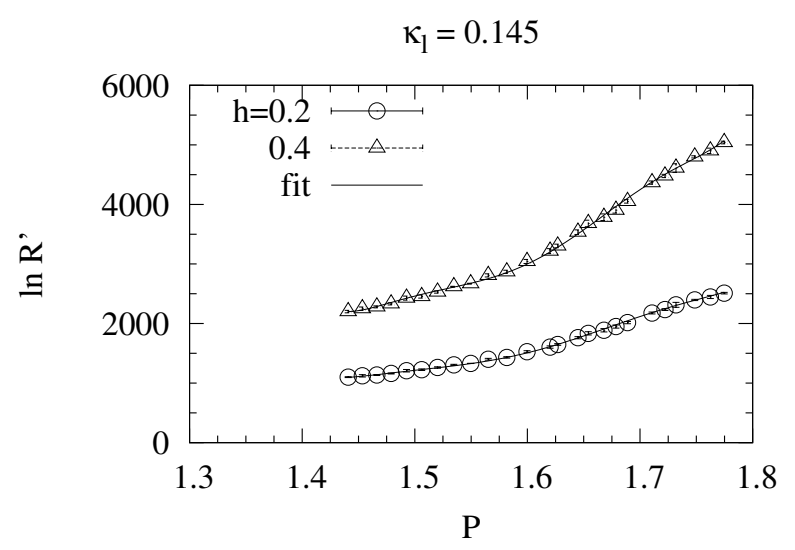

(c)

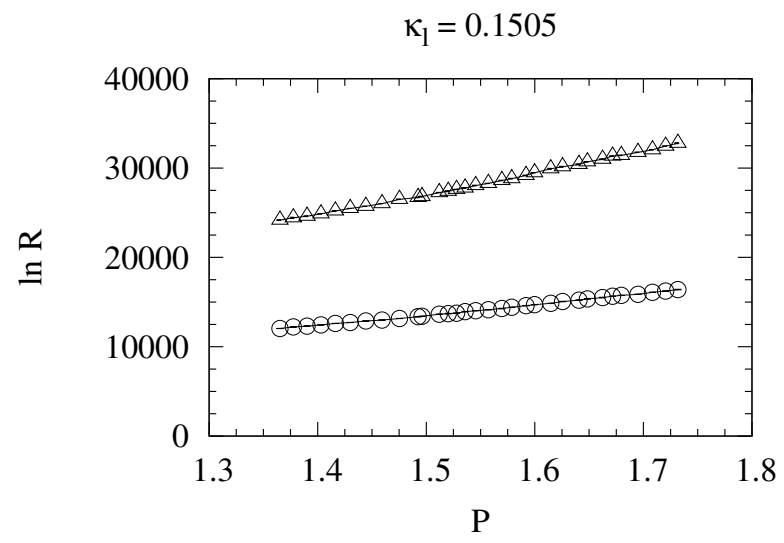

(b)

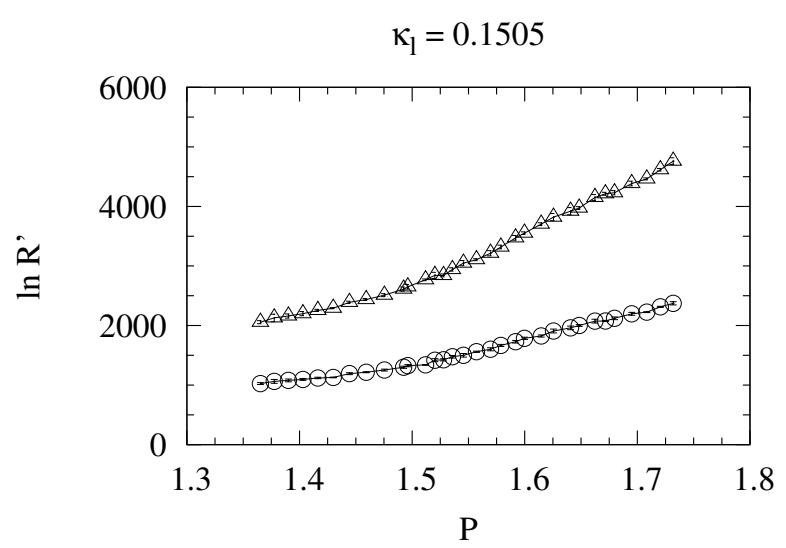

(d)

FIG. 3: $P$ dependence of $\ln R$ [Eq. (18)] at $\kappa_{l}=0.145$ (a) and 0.1505 (b), and $\ln R^{\prime}$ [Eq. (20)] at $\kappa_{l}=0.145(\mathrm{c})$ and $0.1505(\mathrm{~d})$. The results at $h=0.2$ to 0.4 are shown.

$\ln R\left(P ; \kappa_{l}, h\right)$ [Eq. (18)] and $\ln R^{\prime}\left(P ; \kappa_{l}, h\right)$ [Eq. (20)] are calculated. We take two values of $\Delta, 0.0001,0.00025$, to see the stability of the results, and the discrepancy arising from a different choice of $\Delta$ is taken as the systematic uncertainties. In the following plots, the results with $\Delta=0.0001$ are shown unless otherwise stated.

The $P$ dependence of $\ln R\left(P ; \kappa_{l}, h\right)$ and $\ln R^{\prime}\left(P ; \kappa_{l}, h\right)$ are shown in Fig. 3. The statistical errors are invisible on this scale. The sizes of $\ln R$ and $\ln R^{\prime}$ differ by an order of magnitude, which indicates that the difference proportional to $P$ is large and explains why the curvature in $\ln R$ is less clear than that in $\ln R^{\prime}$. The curvature in $\ln R$ and $\ln R^{\prime}$ originates from the rapid increase of the Polyakov loop $(\hat{L})$ contained in Eqs. (16) or $(17)$ around $\beta_{\mathrm{pc}}$.

We then calculate the slope and curvature by fitting the data of $\ln R$ and $\ln R^{\prime}$. Here 
let us mention the fits and the selection of the results. Each data point shown in Fig. 3 is obtained in a separate simulation, and is totally independent of other points. The data points are fit to polynomial functions of $P$ as

$$
\ln R^{()^{\mathrm{fit}}}(P)=\sum_{i=0}^{N_{\text {poly }}} c_{i} P^{i}
$$

over three fit ranges (or three different numbers of the data points), two different polynomial orders, and two values of $\Delta$. Since not all the fits are successful, we only keep the fit results, which satisfy $\chi^{2} /$ dof $<3$, in the following analysis.

Figure 4 shows the values of $\chi^{2} /$ dof obtained from the fit of $\ln R$ and $\ln R^{\prime}$ as a function of the number of the data points used, where only the results with $\chi^{2} /$ dof $<3$ are plotted. It is seen that $\chi^{2} /$ dof with $\Delta=0.00025$ is always larger than that with $\Delta=0.0001$. Once the fit parameters are determined, it is straightforward to calculate the curvature of the potential.

Although we analyze the second derivative to derive the main result, we discuss the first derivative because it is instructive. The first derivative of Eq. (9) is obtained as follows. First, the numerical values of $\bar{P}\left(\beta, \kappa_{l}\right)$ and $\chi_{P}\left(\beta, \kappa_{l}\right)$ are calculated and substituted in Eq. (25) to obtain the two-flavor contribution. Then, the $N_{f}$ flavor's contribution, the first derivative of $\ln R^{\left({ }^{\prime}\right)}$, is determined using the fit results of Eq. (29). By adding up, we obtain the first derivative of the full effective potential. Figure 5 shows the typical behavior of the first derivative of the potential, where the five curves in each plot represent the results for $h=0.0,0.1, \ldots, 0.4$ from top to bottom and the fit results with $\Delta=0.00010$ and $n=5$ are used. It is clear that for $h=0$ the curve is monotonically increasing for all $\kappa_{l}$ while the "S" shape is seen for $h=0.4$. In principle, it may be possible to determine the critical value of $h$ using these plots, however it is not easy to clearly distinguish an "S" shape from a monotonic increase. Thus, we use the second derivative to determine $h_{c}$ as described below.

The results for the curvature are plotted in Fig. 6 for $\ln R$ (solid curves) and $\ln R^{\prime}$ (dashed curves), where the results for $h=0.2$ and 0.4 are shown as examples. Again, the fit results with $\Delta=0.00010$ and $n=5$ are used. The difference in the curvature between $\ln R$ and $\ln R^{\prime}$ turns out to be reasonably small at all $\kappa_{l}$.

Next, the curvature of the first term in Eq. (27) is presented, which can be easily calculated using the averaged value and the susceptibility of $\hat{P}$ at each $\beta$ as in Eq. (26). The curvatures thus obtained are shown in Fig. 6 together with the statistical error, where the fit results 

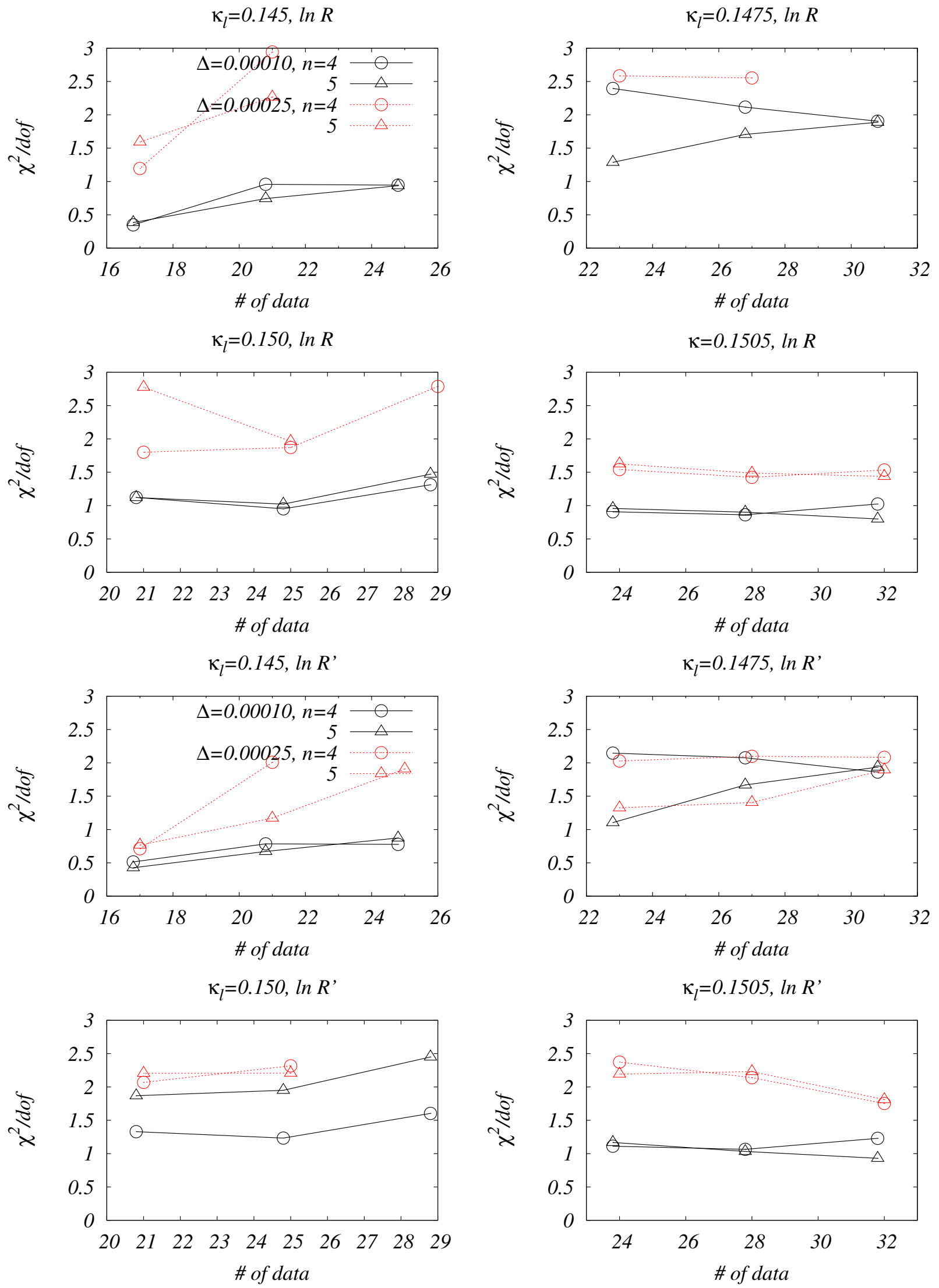

FIG. 4: The resulting $\chi^{2} /$ dof as a function of the number of data points. The results for the $n$-th order polynomial fit are shown. 

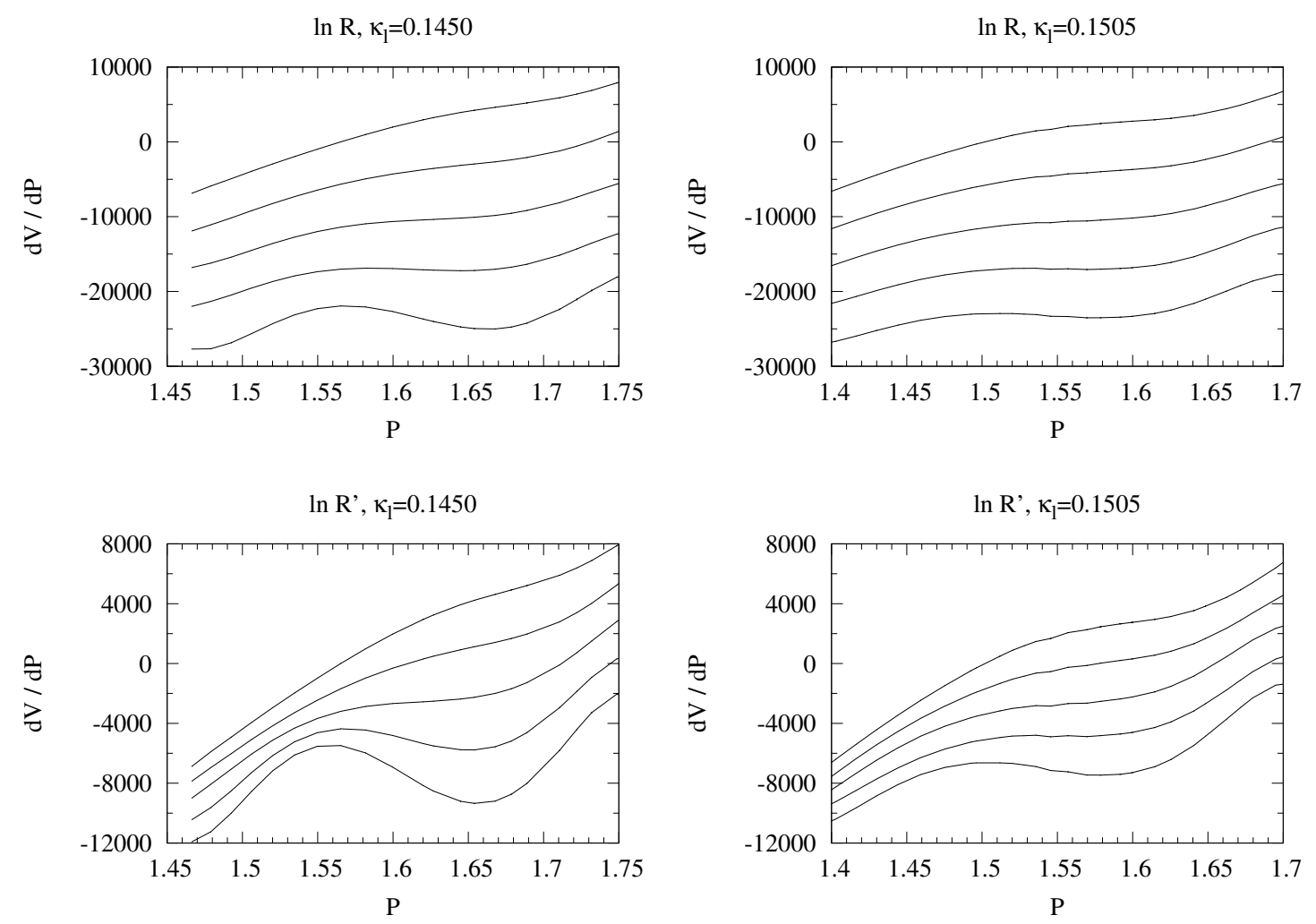

FIG. 5: The first derivative of the full effective potential is shown as a function of $P . h=0.0,0.1$, $0.2,0.3,0.4$ from top to bottom.

obtained with a fifth order of polynomial are shown by dotted curves. It is seen that, independently of $\kappa_{l}, d^{2} V_{\text {light }} / d P^{2}$ is always positive as expected.

Figure 6 shows that $d^{2} \ln R / d P^{2}$ and $d^{2} \ln R^{\prime} / d P^{2}$ have a peak slightly below the $P$ value at which $d^{2} V_{\text {light }} / d P^{2}$ takes the minimum. This indicates that, in the many flavor system, the phase transition or rapid crossover occurs at $P$ smaller than the two-flavor case. For all $\kappa_{l}$, it is observed that the peak of $d^{2} \ln R / d P^{2}$ or $d^{2} \ln R^{\prime} / d P^{2}$ is almost touching the curve of $d^{2} V_{\text {light }} / d P^{2}$ at $h=0.2$, and exceeds $d^{2} V_{\text {light }} / d P^{2}$ at $h=0.4$ in a certain region of $P$.

The resulting curvature of the full effective potentials Eq. (27) for $\kappa_{l}=0.1450$ and 0.1505 are shown in Fig. 7. It is seen that the minimum of the curvature with $h=0.0$ (solid curve) approaches to zero towards the chiral limit of two light flavors. Thus, with this observation alone, one might expect that the potential with $\kappa_{l}=0.1505$ requires only a small $h$ to bring it into the double well shape. However, at $\kappa_{l}=0.1505$, it is also true that adding the heavy quarks does not reduce the minimum of the curvature by much. As a consequence, $h_{c}$ takes a similar value at $\kappa_{l}=0.1450$ and 0.1505 . 

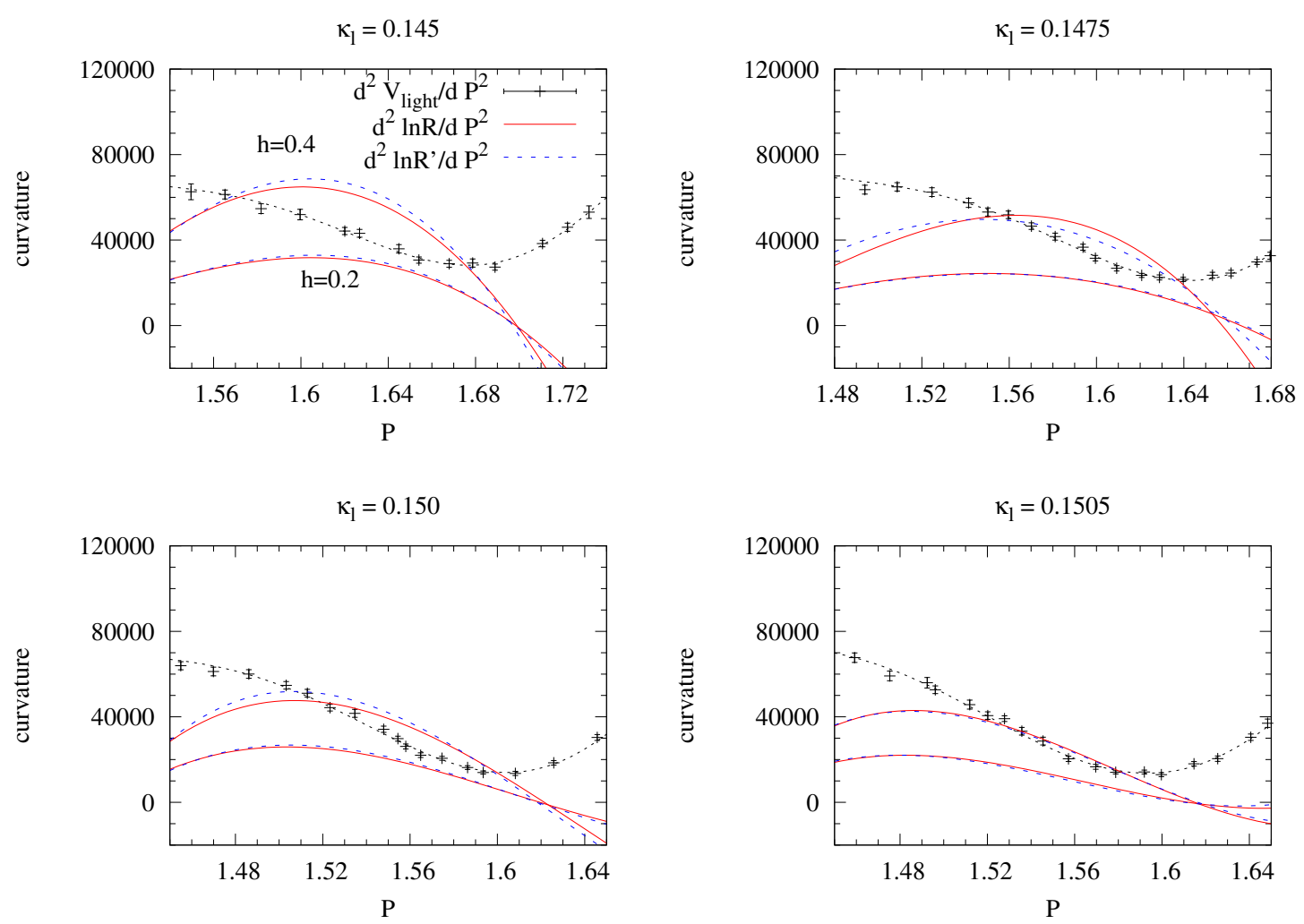

FIG. 6: The second derivative of the first term and the second term of Eq. (27) are shown as a function of $P$. The second term contribution exceeds that of the first term in a range of $P$ when $h=0.4$, which indicates the occurrence of the first order transition at such a value of $h$.

To determine $h_{c}$, we iterate the calculation with $h$ varying in steps of 0.02 . Figure 8 shows the critical values of $h$ as a function of the number of data points used in the fit, corresponding to Fig. 4. Since no reason exists to select the best result from them, we take all the results satisfying $\chi^{2} /$ dof $<3$ as the final results, and the systematic uncertainty is chosen to cover the whole accepted results.

We can also determine the value of $P$ at $h_{c}$, denoted by $P_{c}$ (for the numerical values, see Table II). In Fig. 9, all the results with $\chi^{2} /$ dof $<3$ are plotted together on the $P_{c}-h_{c}$ plane. While $h_{c}$ is insensitive to the two-flavor mass, $P_{c}$ is found to decrease towards the chiral limit of the two-flavor mass. This qualitative feature is tested in the direct simulations of $2+N_{f}$-flavor QCD in Sec. VB.

The light quark mass dependence of $h_{c}$ is plotted in Fig. 10 as a function of the $m_{\pi} / m_{\rho}$ ratio (left) and the PCAC quark mass (right). The error is dominated by the systematic uncertainty associated with the fitting procedure. In the top-right corner of each plot, $h_{c}$ 


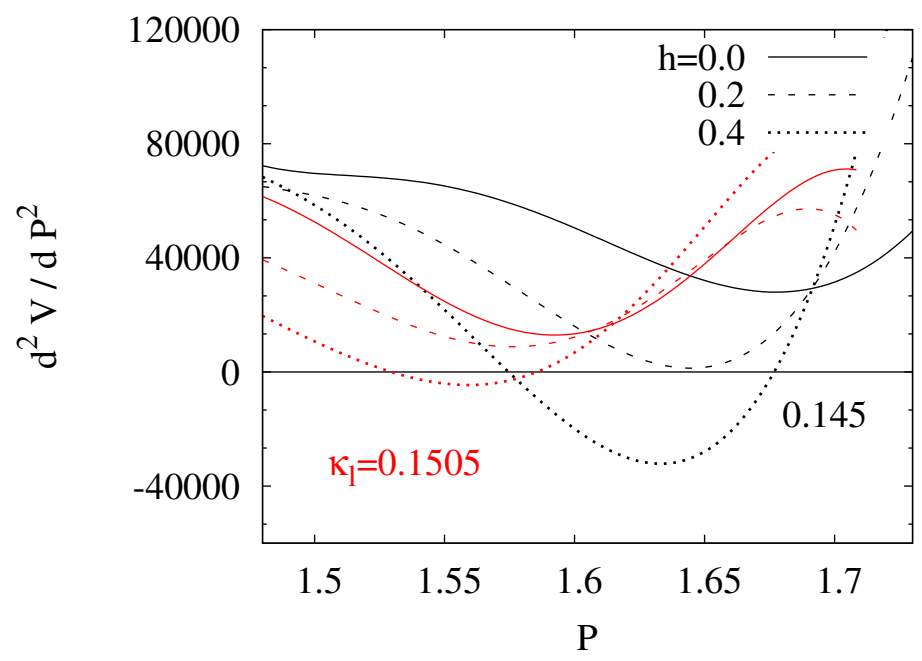

FIG. 7: The curvature of the effective potential at $\kappa_{l}=0.1450$ and 0.1505 and for $h=0.0$ (solid), 0.2 (dashed) and 0.4 (dotted).

\begin{tabular}{c|cc}
$\kappa_{l}$ & $h_{c}$ & $P$ at $h_{c}$ \\
\hline 0.1450 & $0.23(6)$ & $1.627(34)$ \\
0.1475 & $0.27(8)$ & $1.595(27)$ \\
0.1500 & $0.26(5)$ & $1.561(41)$ \\
0.1505 & $0.27(10)$ & $1.572(24)$
\end{tabular}

TABLE II: Numerical results of $h_{c}$ and $P$ at $h_{c}$.

determined from the direct $2+N_{f}$ flavor simulation with $\kappa_{l}=0$ (i.e. $m_{l}=\infty$ ) and $N_{f}=50$ is shown with an uncertainty (for the direct simulations, see Sec. VB). $h_{c}$ at $\kappa_{l}=0$ is clearly larger than those around $0.145 \leq \kappa_{l} \leq 0.1505$, which indicates that $h_{c}$ gradually decreases towards the chiral limit as a global behavior. In the range of $0.145 \leq \kappa_{l} \leq 0.1505$, $h_{c}$ does not show significant dependence on $m_{l}$ within the error, and a constant fit yields $h_{c}=0.23(1)$ in the chiral limit.

If this mild dependence is continued down to the chiral limit and hence $h_{c}$ in the chiral limit remains positive and finite, $h_{c}$ in the chiral limit corresponds to the tricritical point in Fig. 1. Mean field analysis of an effective theory predicts the tricritical scaling [40-43],

$$
\left.h_{c} \sim \text { (const. }\right) \times m_{l}^{2 / 5}+\text { const. }
$$

in the vicinity of the tricritical point, where the power $2 / 5$ is independent of $N_{f}$. In addition to the constant fit (top), we also fit the data to a linear function of $m_{l}^{2 / 5}$ in each plot, yielding 

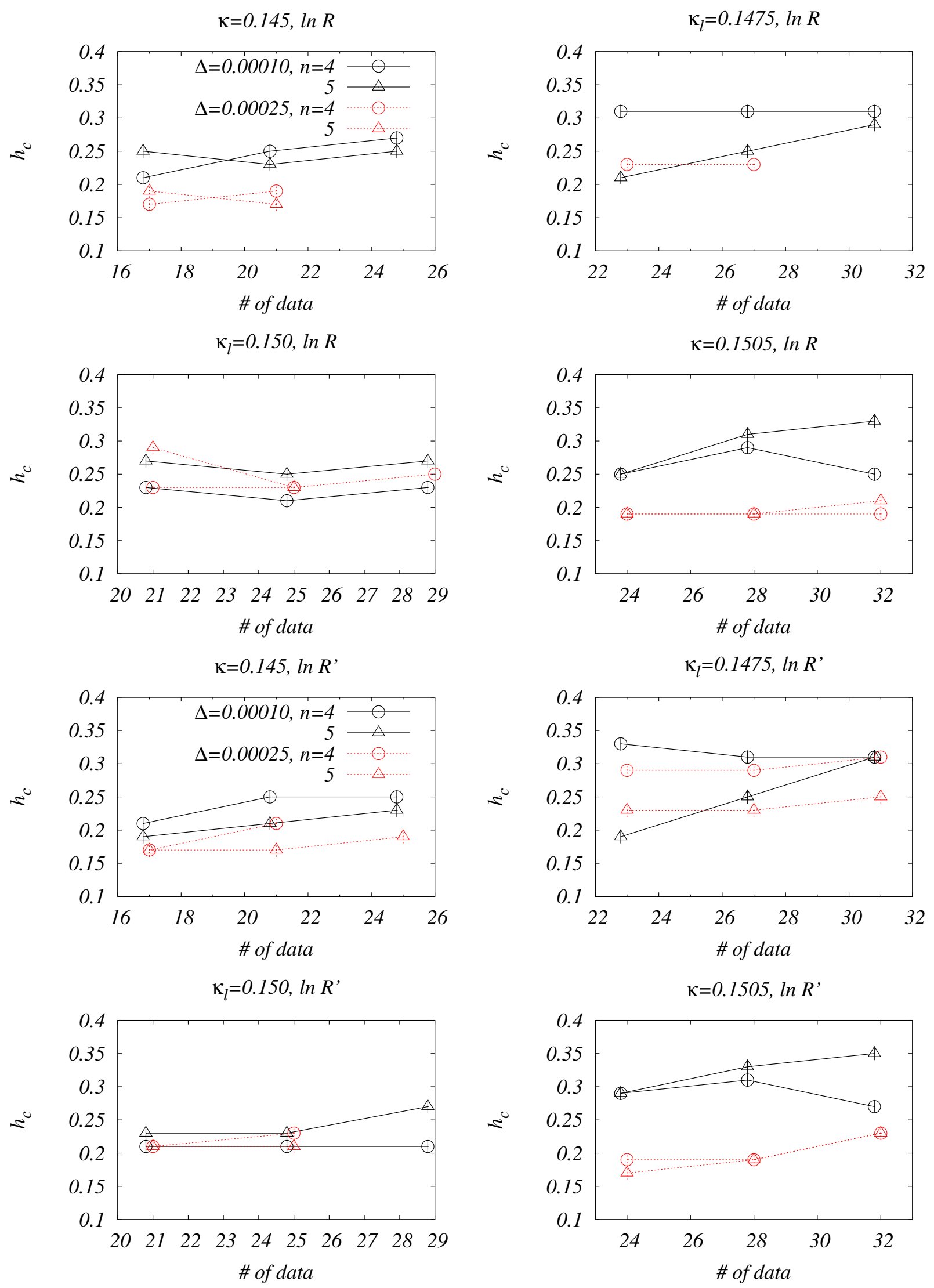

FIG. 8: The critical value of $h$ as a function of the number of the data points used. 


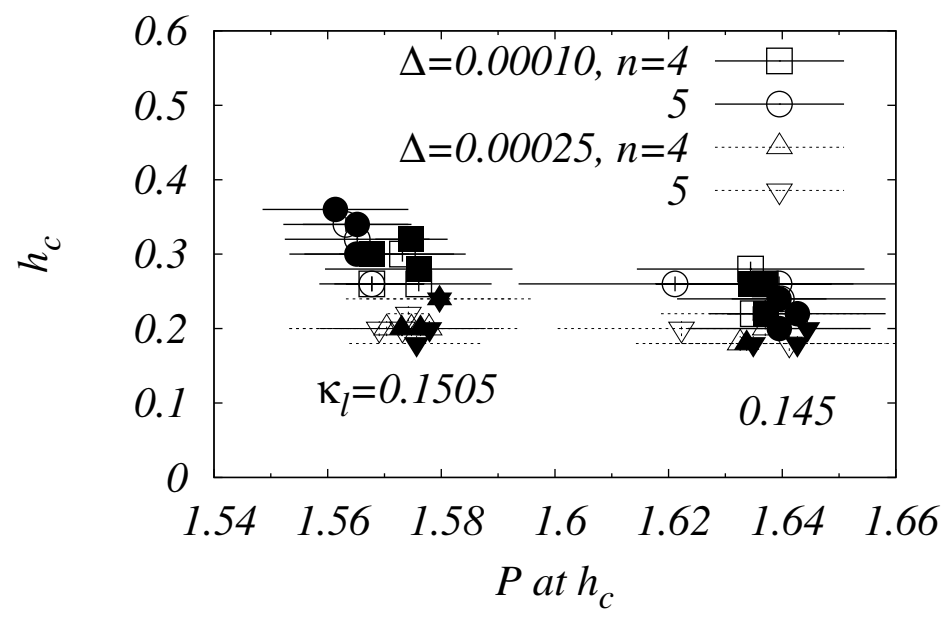

FIG. 9: $h_{c}$ and $P$ at $h_{c}$ : The results from $\ln R$ (open symbols) and $\ln R^{\prime}$ (filled symbols).

$h_{c}=0.35(26)$ and $0.32(17)$ in the chiral limit of the two-flavor mass, respectively. Note that the slope is undetermined and consistent with zero. In either case, a positive value of $h_{c}$ is favored in the chiral limit, which suggests the second order transition of massless two-flavor QCD. Further checks require more extensive lattice calculations and are postponed to future papers.

\section{CONSISTENCY CHECK}

\section{A. Effective potential constraining Polyakov loop}

As an independent check of the results obtained in Sec. IV, we try to estimate $h_{c}$ with a different method. The quantity to be constrained to obtain the PDF is arbitrary as long as it has an overlap with the order parameter. In this section, we take the real part of the Polyakov loop, $\hat{L}$. Recalling Eq. (7), the constraint effective potential for $\hat{L}$ is given by

$$
\begin{aligned}
V_{L}\left(L ; \beta_{\mathrm{ref}}, \kappa_{l}, \kappa_{h}, N_{f}\right) & =-\ln w_{L}\left(L ; \beta_{\mathrm{ref}}, \kappa_{l}, 0,0\right)-\ln \left(\frac{w_{L}\left(L ; \beta_{\mathrm{ref}}, \kappa_{l}, \kappa_{h}, N_{f}\right)}{w_{L}\left(L ; \beta_{\mathrm{ref}}, \kappa_{l}, 0,0\right)}\right) \\
& =V_{L, \text { light }}\left(L ; \beta_{\mathrm{ref}}, \kappa_{l}\right)-\ln R_{L}\left(L ; \beta_{\mathrm{ref}}, \kappa_{l}, \kappa_{h}, N_{f}\right)
\end{aligned}
$$



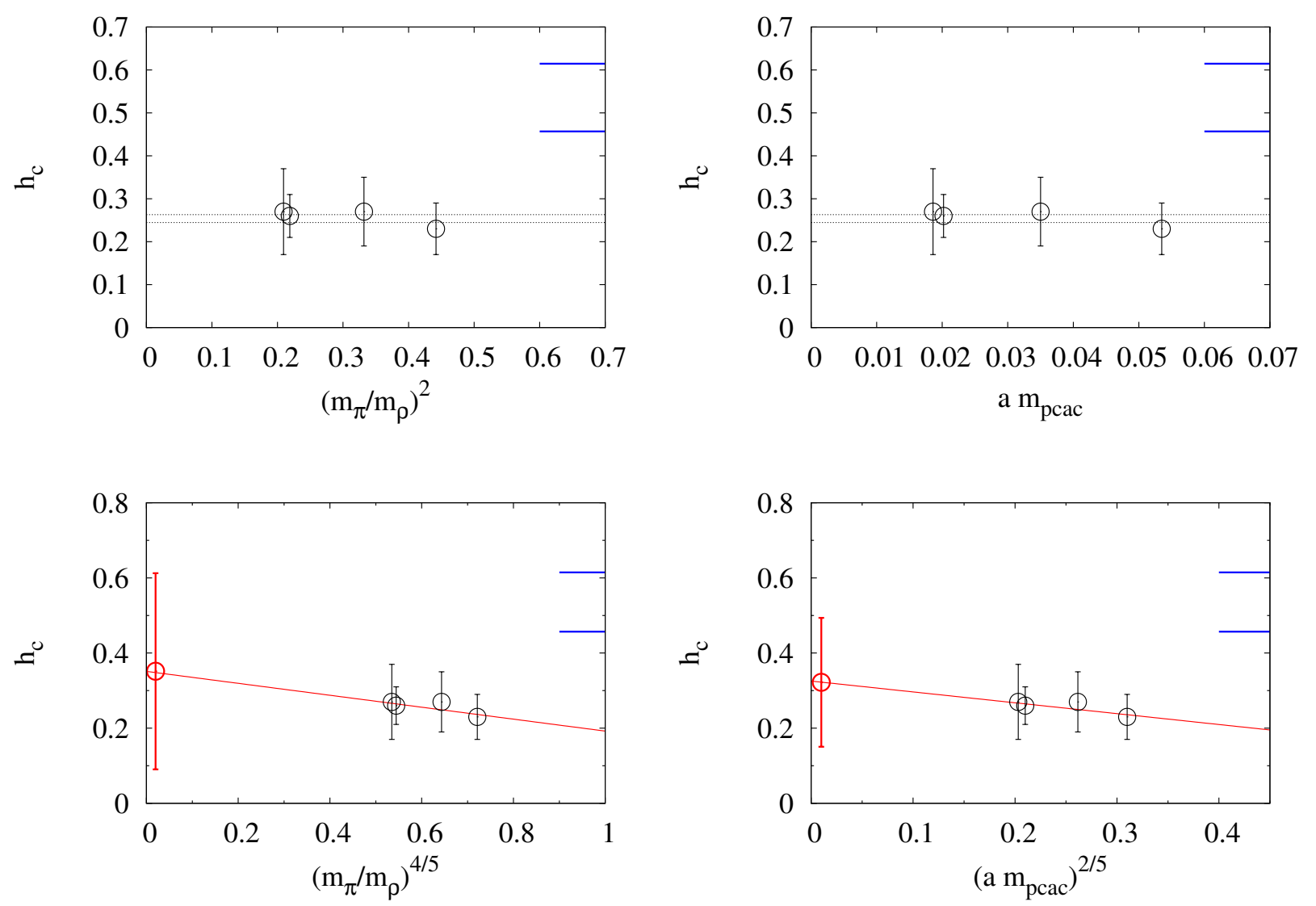

FIG. 10: The light quark mass dependence of $h_{c}$. Top: A constant fit. Bottom: A linear fit is performed with the horizontal axis proportional to $m_{l}^{2 / 5}$, testing the mean field prediction Eq. (30). The band appearing in the top right corner is $h_{c}$ determined in the direct simulation with $\kappa_{l}=0$ and $N_{f}=50$ (for details, see Sec. VB).

where

$$
\begin{aligned}
V_{L, \text { light }}\left(L ; \beta_{\text {ref }}, \kappa_{l}\right) & =-\ln w_{L}\left(L ; \beta_{\text {ref }}, \kappa_{l}, 0,0\right) \\
& =-\ln \left\langle\delta(L-\hat{L}) e^{6\left(\beta_{\text {ref }}-\beta\right) N_{\text {site }} \hat{P}}\right\rangle_{\left(\beta, \kappa_{l}\right)} \\
R_{L}\left(L ; \beta_{\text {ref }}, \kappa_{l}, \kappa_{h}, N_{f}\right) & =e^{6 N_{s}^{3} h L}\left\langle\exp \left[36 N_{s}^{3} h \hat{W}_{P}+6\left(\beta_{\text {ref }}-\beta\right) N_{\text {site }} \hat{P}\right]\right\rangle_{L: \text { fixed },\left(\beta, \kappa_{l}\right)}
\end{aligned}
$$

Unlike the case with $\hat{X}=\hat{P}, \beta_{\text {ref }}$ dependence remains in the second derivative of the potential with respect to $L$, which means $\beta_{\text {ref }}$ has to be explicitly tuned to the (pseudo)critical temperature for each value of $h$. In the crossover region, the minimum of the potential and the minimum of its second derivative are realized at the same value of $L$. On the other hand, in the first order region, $\beta_{\text {ref }}$ has to be tuned until the two minima in the potential 

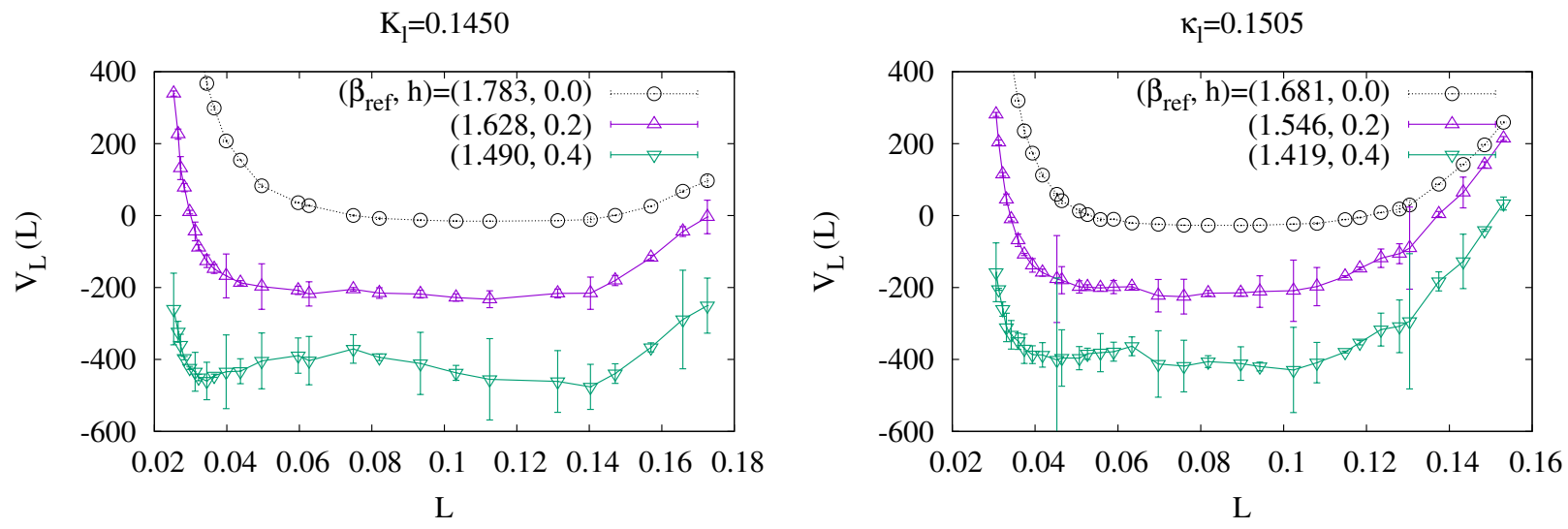

FIG. 11: The effective potential constructed by constraining $\hat{L}$ near the critical temperatures. The absolute values of the potential are shifted for comparison. The results at $h=0.0,0.2$ and 0.4 are shown for $\kappa_{l}=0.145$ (left) and 0.1505 (right).

take the same depth.

The potential, $V_{L}(L)$, is shown in Fig. 11 for $h=0.0,0.2$ and 0.4 , where $V_{L}(L)$ at each $h$ is shifted in a vertical direction for comparison. First of all, the effective potential for the Polyakov loop is not as clean as that for the generalized plaquette, especially at nonzero $h$, which only allows us to extract the qualitative feature. It is seen that the potential has a positive curvature at $h=0$ in our lightest and heaviest light quarks. When $h=0.2$, the potential around the minimum becomes almost flat, indicating that it is close to the end point. If $h$ is further increased to 0.4 , the double well shape appears to emerge though the statistical error makes it ambiguous. These qualitative features are consistent with the findings in the previous section.

\section{B. Direct simulations of $2+N_{f}$ flavor QCD}

Although the convergence of hopping parameter expansion is not the matter for the discussion in the previous section, it is interesting to investigate the convergence of the HPE for future applications. We study this by explicitly performing simulations of $2+N_{f}$ flavor QCD and comparing the results with those based on the HPE. However, thoroughly precise calculations of the many flavor system require an extensive scan of simulation parameters $\left(\kappa_{h}, \beta\right.$ and $\left.N_{f}\right)$ even after fixing $\kappa_{l}$. Furthermore, in general, it is not easy to locate the 
end point of the the first order transition accurately, because the statistical noise grows as one approaches the end point. Instead, we draw a thermal cycle on the $\beta$ - $P$ plane, which is obtained by increasing (or decreasing) $\beta$ until passing its (pseudo) critical value and then reversing the direction. For representative values of $\kappa_{h}, \kappa_{l}$ and $N_{f}$, we accumulated 400 trajectories at each point of the thermal cycle.

We take $\kappa_{l}=0,0.145$ and 0.1505 , and choose $N_{f}$ ranging from 4 to 50 , depending on $\kappa_{l}$, and monitor each thermal cycle whether the hysteresis curve occurs or not. If it occurs, the parameters chosen turn out to be in the first order region. By repeating this, we try to find the critical value of $\kappa_{h}, \kappa_{h_{c}}$, for fixed values of $\kappa_{l}$ and $N_{f}$. The thermal cycles at several simulation parameters are shown in Fig. 12.

Figures 12 (a) and 12 (b) show the thermal cycle at $\kappa_{l}=0$ and $N_{f}=10,16,32,50$, which tells us that $\kappa_{h_{c}}$ decreases with $N_{f}$. Figure 12 (c) shows the same but with nonzero $\kappa_{l}$. Comparing the $N_{f}=16$ data in Figs. 12 (a) and 12 (c) or the $N_{f}=32$ data in Figs. 12 (b) and $12(\mathrm{c})$, it is found that $\kappa_{h_{c}}$ is clearly different between $\kappa_{l}=0$ and 0.1505 . In Fig. 9 of Sec. IV A, we have discussed that the HPE predicts that $P$ at $h_{c}$ decreases with $\kappa_{l}$. Figure 12 (d) seems to show that it is the case, at least, qualitatively.

The critical values, $\kappa_{h_{c}}$, obtained at each $\kappa_{l}$ and $N_{f}$ are translated into $h_{c}$ using Eq. (15), and plotted as a function of $N_{f}$ in Fig. 13. It is expected that, for a sufficiently large $N_{f}$, $h_{c}$ approaches an asymptotic value, but the value of $N_{f}$ we have studied seems not to reach such a region yet although the increasing rate looks slowing down. Importantly, it is seen that $\kappa_{h_{c}}$ does not differ by much between $\kappa_{l}=0.145$ and 0.1505 , which is consistent with the observation in the HPE analysis. It is interesting to include the next-to-leading order contribution of the HPE analysis.

\section{SUMMARY AND DISCUSSION}

We have studied the finite temperature phase transition of QCD with two light and many heavy quarks at zero chemical potential, where the heavy quarks are introduced in the form of the hopping parameter expansion via the reweighting method. The phase structure was scanned on the $\kappa_{l}$ - $h$ plane to identify the critical line separating the continuous crossover and the first order regions.

The nature of the transition is identified by the shape of the constraint effective potential 


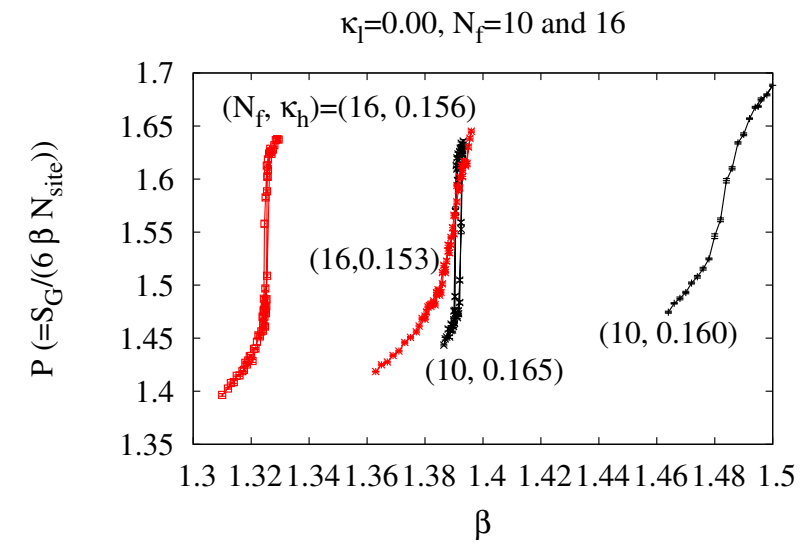

(a)

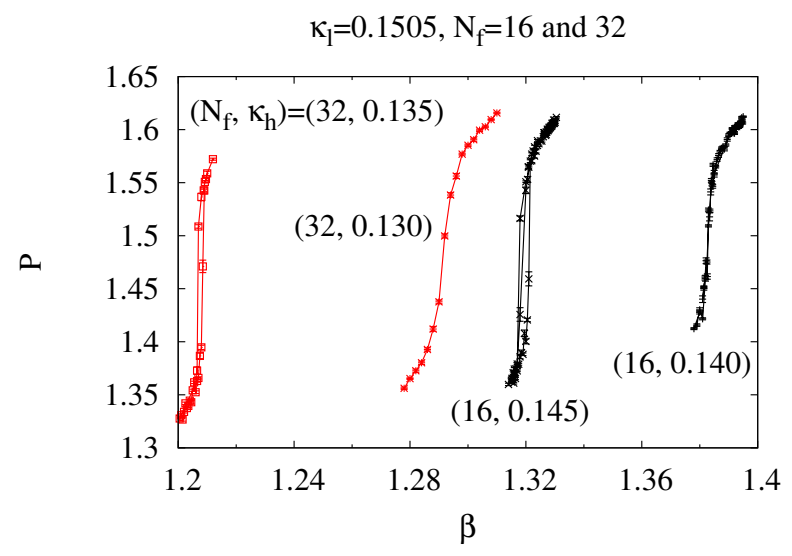

(c)

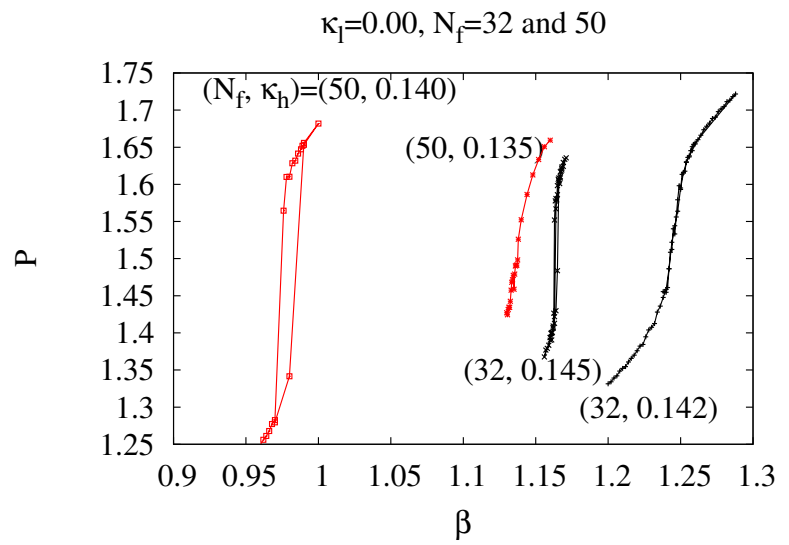

(b)

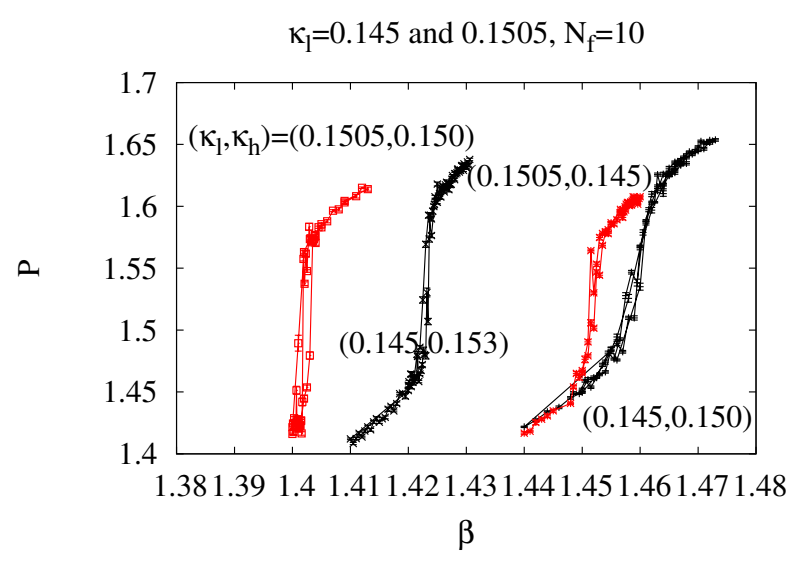

(d)

FIG. 12: The thermal cycle on the $(P, \beta)$-plane in $2+N_{f}$ flavor QCD. The vicinity of the end point, i.e. $\kappa_{h} \approx \kappa_{h_{c}}$, is shown.

constructed from the probability distribution function of the generalized plaquette. For $h=0$, the system reduces to two-flavor QCD, which always shows continuous crossover and hence the potential has a single well. As one increases $h$, at some point the potential takes a double-well shape, which defines a critical value, $h_{c}$. We have determined $h_{c}$ at four light quark masses, and observed that $h_{c}$ is independent of two-flavor mass in the range we have studied $\left(0.46 \leq m_{\pi} / m_{\rho} \leq 0.66\right)$. This result indicates that the critical heavy mass remains finite in the chiral limit of the two flavors, suggesting the phase transition of massless twoflavor QCD is of second order. Some of the qualitative features observed in the main analysis were checked by two independent analyses. 


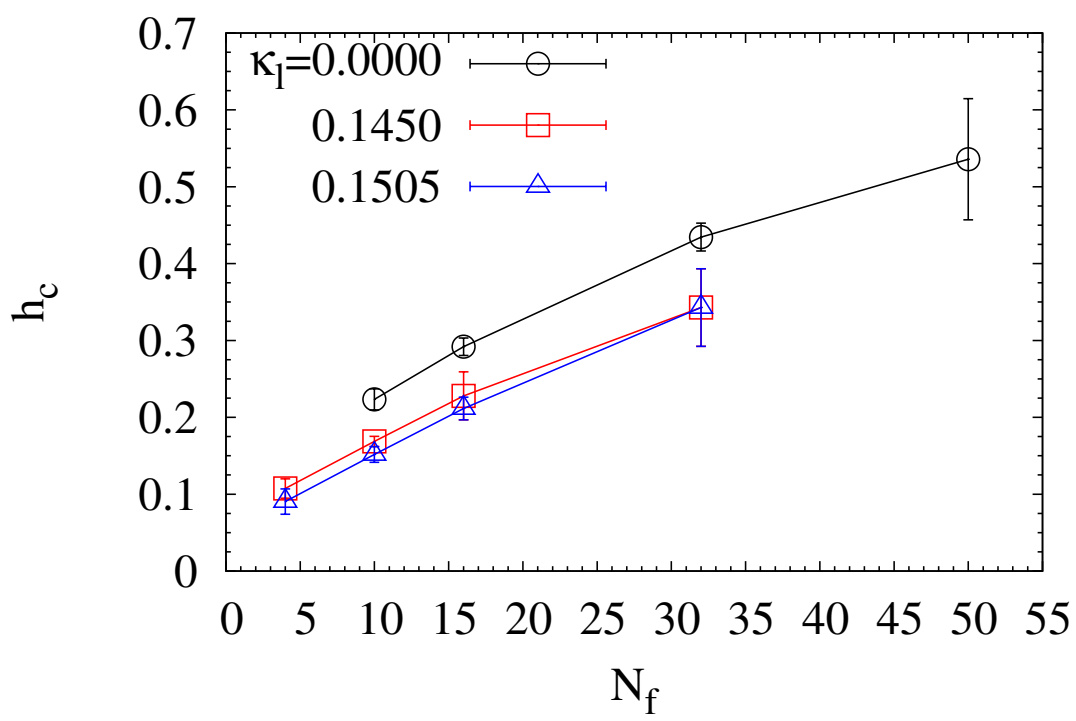

FIG. 13: $N_{f}$ dependence of $h_{c}$ through direct simulations of $2+N_{f}$ flavor QCD.

The approach in this study can be said as follows. Two-flavor QCD with a finite mass is enforced to undergo a first order phase transition by adding extra quarks. It is then likely that those extra quarks are necessary to keep the first order transition down to the chiral limit of two-flavor QCD. This method is applicable for any kinds of lattice fermions unless they contain $\beta$ dependent coefficients. According to the definition of $h$, Eq. (15), $\kappa_{h_{c}}$ can be considered to be arbitrarily small for a given $h_{c}$ by assuming arbitrarily large $N_{f}$, and thus we do not have to care about the convergence of the hopping parameter expansion. Nevertheless, it is interesting to see the limitation of the HPE for a fixed $N_{f}$ for further applications.

In order to establish our finding, possible systematic uncertainties, which are not investigated in the present paper, need to be understood. Since, at the end point, the second order phase transition occurs, a sizable finite volume effect is possible in the vicinity of the point. However, we do not expect it to be significant in the many flavor approach. In this approach, the end point is determined through the extrapolation of the effective potential with regard to $h$, and the extrapolation is performed in a region free from finite size effects since the two-flavor configurations are all generated at a parameter region away from the second order end point. Namely, at the price of the uncertainty due to the extrapolation, we could have avoided the finite volume effect associated with the second order phase transition. 
Nevertheless, it is clearly important to explicitly check that the effect is under good control. Such work is ongoing.

Although the behavior observed in Fig. 10 seems to suggest that the chiral limit of $h_{c}$ is finite and positive, it then has to show the tricritical scaling [Eq. (30)]. At the present, our results allow us to fit to any smooth function in $m_{l}$. In order to improve the situation, we need to explore lighter quark masses and reduce the systematic uncertainty associated with the fitting procedure. However, during the preliminary study, we realized that the lightest quark mass presented in this paper is the lower limit in our lattice setup. To go beyond the limit, the setup has to be changed. Towards the ultimate goal, the discretization effects also have to be examined. A systematic study of these uncertainties requires large scale simulations, and we postpone them to future works.

We can extend the many flavor approach to explore QCD at finite chemical potential as initiated in Refs. [24, 44]. In this case, mean field analysis predicts that the critical line runs like $m_{l}^{c} \sim|\mu|^{5}[43]$. We believe that such a study brings valuable information to understand the rich QCD phase diagram.

\section{Acknowledgments}

We would like to thank members of the WHOT-QCD Collaboration for useful discussions. We also thank Ken-Ichi Ishikawa for providing us his simulation codes. This work is in part supported by JSPS KAKENHI Grant-in-Aid for Scientific Research (B) (No. $15 \mathrm{H} 03669$ [N. Y.], 26287040 [S. E.] ) and (C) (No. 26400244 [S. E.]), and by the Large Scale Simulation Program of High Energy Accelerator Research Organization (KEK) No. 14/15-23.

[1] R. D. Pisarski and F. Wilczek, "Remarks on the Chiral Phase Transition in Chromodynamics," Phys. Rev. D 29, 338 (1984).

[2] A. Butti, A. Pelissetto, and E. Vicari, "On the nature of the finite temperature transition in QCD," JHEP 0308, 029 (2003) [hep-ph/0307036].

[3] S. Aoki, H. Fukaya and Y. Taniguchi, "Chiral symmetry restoration, eigenvalue density of Dirac operator and axial U(1) anomaly at finite temperature," Phys. Rev. D 86 (2012) 114512 [arXiv: 1209.2061 [hep-lat]]. 
[4] A. Pelissetto and E. Vicari, "Relevance of the axial anomaly at the finite-temperature chiral transition in QCD," Phys. Rev. D 88, 105018 (2013) [arXiv:1309.5446 [hep-lat]].

[5] Y. Nakayama and T. Ohtsuki, "Bootstrapping phase transitions in QCD and frustrated spin systems,", Phys. Rev. D 91, 021901 (2015) arXiv:1407.6195 [hep-th].

[6] T. Sato and N. Yamada, "Linking $U(2) \times U(2)$ to $O(4)$ model via decoupling," Phys. Rev. D 91, 034025 (2015) [arXiv:1412.8026 [hep-lat]].

[7] F. Karsch, "Scaling of pseudocritical couplings in two flavor QCD," Phys. Rev. D 49, 3791 (1994) [hep-lat/9309022].

[8] F. Karsch and E. Laermann, "Susceptibilities, the specific heat and a cumulant in two flavor QCD," Phys. Rev. D 50, 6954 (1994) [hep-lat/9406008].

[9] Y. Iwasaki, K. Kanaya, S. Kaya, and T. Yoshie, "Scaling of chiral order parameter in two flavor QCD," Phys. Rev. Lett. 78, 179 (1997) [hep-lat/9609022].

[10] A. Ali Khan et al. [CP-PACS Collaboration], "Phase structure and critical temperature of two flavor QCD with renormalization group improved gauge action and clover improved Wilson quark action," Phys. Rev. D 63, 034502 (2001) [hep-lat/0008011].

[11] M. D'Elia, A. Di Giacomo, and C. Pica, "Two flavor QCD and confinement," Phys. Rev. D 72 (2005) 114510 [hep-lat/0503030].

[12] C. Bonati, G. Cossu, M. D'Elia, A. Di Giacomo, and C. Pica, "A Test of first order scaling in Nf =2 QCD: A Progress report," PoS LATTICE 2008, 204 (2008) [arXiv:0901.3231 [hep-lat]].

[13] S. Ejiri, F. Karsch, E. Laermann, C. Miao, S. Mukherjee, P. Petreczky, C. Schmidt, W. Soeldner, and W. Unger, "Magnetic equation of state in (2+1)-flavor QCD," Phys. Rev. D 80, 094505 (2009).

[14] For a review concerning this topic, see, for example, K. Kanaya, "Finite Temperature QCD on the Lattice - Status 2010," PoS LATTICE 2010, 012 (2010) [arXiv: 1012.4247 [hep-lat]].

[15] C. Bonati, P. de Forcrand, M. D'Elia, O. Philipsen, and F. Sanfilippo, "Chiral phase transition in two-flavor QCD from an imaginary chemical potential," Phys. Rev. D 90, 074030 (2014) [arXiv: 1408.5086 [hep-lat]].

[16] B. B. Brandt, A. Francis, H. B. Meyer, O. Philipsen, and H. Wittig, "QCD thermodynamics with $\mathrm{O}(\mathrm{a})$ improved Wilson fermions at $N_{f}=2$," PoS LATTICE 2013, 162 (2014) [arXiv: 1310.8326 [hep-lat]]. 
[17] G. Cossu, S. Aoki, H. Fukaya, S. Hashimoto, T. Kaneko, H. Matsufuru, and J. I. Noaki, "Finite temperature study of the axial U(1) symmetry on the lattice with overlap fermion formulation," Phys. Rev. D 87, 114514 (2013) [Phys. Rev. D 88, 019901 (2013)] [arXiv: 1304.6145 [hep-lat]].

[18] M. I. Buchoff, M. Cheng, N. H. Christ, H.-T. Ding, C. Jung, F. Karsch, Z. Lin, R. D. Mawhinney et al., "QCD chiral transition, U(1)A symmetry and the dirac spectrum using domain wall fermions," Phys. Rev. D 89, 054514 (2014) [arXiv: 1309.4149 [hep-lat]].

[19] T. Bhattacharya, M. I. Buchoff, N. H. Christ, H.-T. Ding, R. Gupta, C. Jung, F. Karsch, Z. Lin et al., "The QCD phase transition with physical-mass, chiral quarks," Phys. Rev. Lett. 113, 082001 (2014) [arXiv: 1402.5175 [hep-lat]].

[20] V. Dick, F. Karsch, E. Laermann, S. Mukherjee, and S. Sharma, "Microscopic origin of $U_{A}(1)$ symmetry violation in the high temperature phase of QCD," Phys. Rev. D 91, 094504 (2015) [arXiv: 1502.06190 [hep-lat]].

[21] R. Kitano and N. Yamada, "Topology in QCD and the axion abundance," JHEP 1510, 136 (2015) [arXiv: 1506.00370 [hep-ph]].

[22] E. Berkowitz, M. I. Buchoff and E. Rinaldi, "Lattice QCD input for axion cosmology," Phys. Rev. D 92, no. 3, 034507 (2015) [arXiv:1505.07455 [hep-ph]].

[23] S. Borsanyi et al., "Axion cosmology, lattice QCD and the dilute instanton gas," Phys. Lett. B 752, 175 (2016) [arXiv:1508.06917 [hep-lat]].

[24] S. Ejiri and N. Yamada, "End Point of a First-Order Phase Transition in Many-Flavor Lattice QCD at Finite Temperature and Density," Phys. Rev. Lett. 110, 172001 (2013) [arXiv: 1212.5899 [hep-lat]].

[25] S. Ejiri, N. Yamada, and H. Yoneyama, "First order transition regions in the quark masses and chemical potential parameter space of QCD," arXiv:1504.02283 [hep-lat].

[26] F. R. Brown, F. P. Butler, H. Chen, N. H. Christ, Z. h. Dong, W. Schaffer, L. I. Unger, and A. Vaccarino, "On the existence of a phase transition for QCD with three light quarks," Phys. Rev. Lett. 65, 2491 (1990).

[27] Y. Aoki, G. Endrodi, Z. Fodor, S. D. Katz, and K. K. Szabo, "The Order of the quantum chromodynamics transition predicted by the standard model of particle physics," Nature $\mathbf{4 4 3}$, 675 (2006) [hep-lat/0611014].

[28] H.-T. Ding, A. Bazavov, F. Karsch, Y. Maezawa, S. Mukherjee, and P. Petreczky, "Chiral 
phase transition of $N_{f}=2+1$ QCD with the HISQ action," PoS LATTICE 2013, 157 (2014) [arXiv:1312.0119 [hep-lat]].

[29] X. Y. Jin, Y. Kuramashi, Y. Nakamura, S. Takeda, and A. Ukawa, "Critical endpoint of the finite temperature phase transition for three flavor QCD," Phys. Rev. D 91, no. 1, 014508 (2015) [arXiv: 1411.7461 [hep-lat]].

[30] C.R. Allton, S. Ejiri, S.J. Hands, O. Kaczmarek, F. Karsch, E. Laermann, Ch. Schmidt, and L. Scorzato, "QCD thermal phase transition in the presence of a small chemical potential," Phys. Rev. D 66, 074507 (2002).

[31] S. Ejiri, C.R. Allton, S.J. Hands, O. Kaczmarek, F. Karsch, E. Laermann, and Ch. Schmidt, "Study of QCD thermodynamics at finite density by Taylor expansion," Prog. Theor. Phys. Suppl. 153, 118 (2004).

[32] A. D. Bruce, "Probability density functions for collective coordinates in Ising-like systems", J. Phys. C: Solid State Phys. 14 (1981) 3667.

[33] K. Binder, "Finite size scaling analysis of Ising model block distribution functions," Z. Phys. B 43, 119 (1981).

[34] For a brief review, see, for example, J. A. Plascak and P. H. L. Martins, "Probability distribution function of the order parameter: Mixing fields and universality," Comput. Phys. Commun. 184, 259 (2013).

[35] H. Saito, S. Ejiri, S. Aoki, T. Hatsuda, K. Kanaya, Y. Maezawa, H. Ohno, and T. Umeda (WHOT-QCD Collaboration), "Phase structure of finite temperature QCD in the heavy quark region," Phys. Rev. D 84, 054502 (2011). [arXiv: 1106.0974].

[36] S. Ejiri, "On the existence of the critical point in finite density lattice QCD," Phys. Rev. D 77, 014508 (2008) [arXiv:0706.3549 [hep-lat]].

[37] S. Ejiri, "Phase structure of hot dense QCD by a histogram method," Eur. Phys. J. A 49, 86 (2013) [arXiv: 1306.0295 [hep-lat]].

[38] S. Ejiri, "Canonical partition function and finite density phase transition in lattice QCD," Phys. Rev. D 78, 074507 (2008) [arXiv:0804.3227 [hep-lat]].

[39] S. Ejiri, Y. Maezawa, N. Ukita, S. Aoki, T. Hatsuda, N. Ishii, K. Kanaya, and T. Umeda (WHOT-QCD Collaboration), "Equation of State and Heavy-Quark Free Energy at Finite Temperature and Density in Two Flavor Lattice QCD with Wilson Quark Action," Phys. Rev. D 82, 014508 (2010). 
[40] F. Wilczek, "Application of the renormalization group to a second order QCD phase transition," Int. J. Mod. Phys. A 7, 3911 (1992); 7, 6951 (1992)].

[41] K. Rajagopal and F. Wilczek, "Static and dynamic critical phenomena at a second order QCD phase transition," Nucl. Phys. B 399, 395 (1993) [hep-ph/9210253].

[42] A. Ukawa, "Lectures on lattice QCD at finite temperature," UTHEP-302, C93-06-21.1.

[43] S. Ejiri, "Recent progress in lattice QCD at finite density," PoS LATTICE 2008, 002 (2008) [arXiv:0812.1534 [hep-lat]].

[44] R. Iwami, S. Ejiri and N. Yamada, "Many flavor approach to study the critical point in finite density QCD," arXiv:1512.08317 [hep-lat]. 